\title{
Vibrational Spectroscopy and Analysis of Pseudo-tetrahedral Complexes with Metal Imido Bonds
}

\author{
Mark P. Mehn, ${ }^{\dagger}{ }^{\ddagger}$ Steven D. Brown, ${ }^{\ddagger}$ David M. Jenkins, ${ }^{\ddagger}$ Jonas C. Peters, ${ }^{\ddagger \star}$ Lawrence \\ Que, Jr. ${ }^{+*}$ \\ Department of Chemistry and Center for Metals in Biocatalysis, 207 Pleasant St. S.E., University of \\ Minnesota, Minneapolis, Minnesota 55455, U.S.A. and \\ Department of Chemistry and Chemical Engineering, Arnold and Mabel Beckman Laboratories of \\ Chemical Synthesis, California Institute of Technology, Pasadena, California 91125, U.S.A.
}

\section{Supporting Information}

Details for crystallographic studies and structural refinement

Table S1. Crystal data and structural refinement details for $\left[\mathrm{PhBP}_{3}\right] \mathrm{Fe} \equiv \mathrm{N}^{t} \mathrm{Bu}$ and $\left[\mathrm{PhBP}_{3}\right] \mathrm{Co} \equiv \mathrm{N}^{t} \mathrm{Bu}$

Figure S1. Fully labeled drawing of $\left[\mathrm{PhBP}_{3}\right] \mathrm{Fe} \equiv \mathrm{N}^{t} \mathrm{Bu}$

Table S2. Atomic coordinates and equivalent isotropic displacement parameters for $\left[\mathrm{PhBP}_{3}\right] \mathrm{Fe} \equiv \mathrm{N}^{t} \mathrm{Bu}$

Table S3. Bond lengths and angles for $\left[\mathrm{PhBP}_{3}\right] \mathrm{Fe} \equiv \mathrm{N}^{t} \mathrm{Bu}$

Table S4. Anisotropic parameters for $\left[\mathrm{PhBP}_{3}\right] \mathrm{Fe} \equiv \mathrm{N}^{t} \mathrm{Bu}$

Table S5. Hydrogen coordinates and isotropic displacement parameters for $\left[\mathrm{PhBP}_{3}\right] \mathrm{Fe} \equiv \mathrm{N}^{t} \mathrm{Bu}$

Figure S2. Fully labeled drawing of $\left[\mathrm{PhBP}_{3}\right] \mathrm{Co} \equiv \mathrm{N}^{t} \mathrm{Bu}$

Table S6. Atomic coordinates ( $\times 10^{4}$ ) and equivalent isotropic displacement parameters $\left(\AA^{2} \mathrm{x} 10^{3}\right)$ for $\left[\mathrm{PhBP}_{3}\right] \mathrm{Co} \equiv \mathrm{N}^{t} \mathrm{Bu}$

Table S7. Bond lengths and angles for $\left[\mathrm{PhBP}_{3}\right] \mathrm{Co} \equiv \mathrm{N}^{t} \mathrm{Bu}$

Table S8. Anisotropic parameters for $\left[\mathrm{PhBP}_{3}\right] \mathrm{Co} \equiv \mathrm{N}^{t} \mathrm{Bu}$

Table S9. Hydrogen coordinates and isotropic displacement parameters for $\left[\mathrm{PhBP}_{3}\right] \mathrm{Co} \equiv \mathrm{N}^{t} \mathrm{Bu}$ 
Details for crystallographic studies and structural refinement. Diffraction of crystals of $\left[\mathrm{PhBP}_{3}\right] \mathrm{Fe} \equiv \mathrm{N}^{t} \mathrm{Bu}$ and $\left[\mathrm{PhBP}_{3}\right] \mathrm{Co} \equiv \mathrm{N}^{t} \mathrm{Bu}$ were carried out in the Beckman Institute Crystallographic Facility at Caltech on a Bruker Smart 1000 CCD diffractometer using graphite-monochromated $\operatorname{MoK} \alpha(\lambda=0.71073 \AA)$ radiation. The crystals were mounted on a glass fiber with Paratone N oil. A summary of the crystallographic details for both crystals is given in Table $\mathrm{S} 1$. For $\left[\mathrm{PhBP}_{3}\right] \mathrm{Fe}=\mathrm{N}^{t} \mathrm{Bu}$, the crystal is twinned by a 180 degree rotation about the $\mathrm{c}^{*}$ axis. The major to minor component ratio refined to 53:47. Since the crystal was a non-merohedral twin crystal, there is more than one entry in the reflection file for a given set of $\mathrm{h}, \mathrm{k}, \mathrm{l}$ indices (i.e. data read into SHELXL with HKLF 5). As a result, more reflections were used in the refinement than the apparent number of unique reflections. All non-hydrogen atoms were refined anisotropically. The final full matrix least squares refinement converged to $R 1=0.0429$ and $w R 2=0.0886$ for $\left[\mathrm{PhBP}_{3}\right] \mathrm{Fe} \equiv \mathrm{N}^{t} \mathrm{Bu}$ and $R 1=0.0401$ and $w R 2=0.0690$ for $\left[\mathrm{PhBP}_{3}\right] \mathrm{Co} \equiv \mathrm{N}^{t} \mathrm{Bu}$. Crystallographic data for $\left[\mathrm{PhBP}_{3}\right] \mathrm{Co} \equiv \mathrm{N}^{t} \mathrm{Bu}$ have been deposited at the CCDC, 12 Union Road, Cambridge CB2 1EZ, UK and copies can be obtained on request, free of charge, by quoting the publication citation and the deposition number 197202 . 
Table S1. Summary of Crystallographic Data $\left[\mathrm{PhBP}_{3}\right] \mathrm{Fe} \equiv \mathrm{N}^{t} \mathrm{Bu}$ and $\left[\mathrm{PhBP}_{3}\right] \mathrm{Co} \equiv \mathrm{N}^{t} \mathrm{Bu}$

\begin{tabular}{lll}
\hline & \multicolumn{1}{c}{$\left[\mathrm{PhBP}_{3}\right] \mathrm{Fe} \equiv \mathrm{N}^{t} \mathrm{Bu}$} & \multicolumn{1}{c}{$\left[\mathrm{PhBP}_{3}\right\rceil \mathrm{Co} \equiv \mathrm{N}^{t} \mathrm{Bu}$} \\
\hline empirical formula & $\mathrm{C}_{49} \mathrm{H}_{50} \mathrm{BFeNP}_{3}$ & $\mathrm{C}_{49} \mathrm{H}_{50} \mathrm{BCoNP}_{3} \cdot 1.5 \mathrm{C}_{6} \mathrm{H}_{6}$ \\
formula weight $(\mathrm{g} / \mathrm{mol})$ & 812.47 & 932.71 \\
crystal habit, color & chunk, deep red & chunk, deep red \\
crystal system & monoclinic & monoclinic \\
space group & $P{ }_{1} / n(\# 14)$ & $P 2_{1} / n(\# 14)$ \\
$a(\AA)$ & $14.7124(13)$ & $11.2939(5)$ \\
$b(\AA)$ & $19.0114(17)$ & $18.4709(8)$ \\
$c(\AA)$ & $15.0887(13)$ & $23.6972(11)$ \\
$\alpha$, deg & 90 & 90 \\
$\beta$, deg & $96.9378(17)$ & $99.9680(10)$ \\
$\gamma$, deg & 90 & 90 \\
$V\left(\AA^{3}\right)$ & $4189.5(6)$ & $4868.8(4)$ \\
$Z$ & 4 & 4 \\
$D_{\text {calc }}\left(\mathrm{g} / \mathrm{cm}{ }^{3}\right)$ & 1.288 & 1.272 \\
Temperature $(\mathrm{K})$ & 98 & 98 \\
Absorption $\left(\mathrm{cm}{ }^{-1}\right)$ & 5.10 & 4.82 \\
$\theta$ range $($ deg $)$ & $1.73-28.84$ & $2.15-28.43$ \\
$h k l$ ranges & $-19-19,0-25,0-20$ & $-14-15,-24-24,-30-31$ \\
no. of reflections collected & 21848 & 71545 \\
no. of unique reflections & $21954\left(\mathrm{R}_{\text {int }}=\mathrm{N} . \mathrm{A}.\right)$ & $11520\left(\mathrm{R}_{\text {int }}=0.0607\right)$ \\
observed reflections $(I>2 \sigma(I))$ & 16756 & 8539 \\
Parameters & 500 & 580 \\
$\mathrm{R} 1 /$ wR2 $(I>2 \sigma(I))$ & $0.0429 / 0.0848$ & $0.0401 / 0.0669$ \\
$\mathrm{R} 1 /$ wR2 $($ all data $)$ & $0.0651 / 0.0886$ & $0.0626 / 0.0690$ \\
goodness-of-fit on $F^{2}$ & 1.614 & 1.794 \\
max/min peak, e/ $\AA^{3}$ & $0.584 /-0.434$ & $0.636 /-0.488$ \\
\hline
\end{tabular}


Figure S1. Fully labeled drawing of $\left[\mathrm{PhBP}_{3}\right] \mathrm{Fe} \equiv \mathrm{N}^{t} \mathrm{Bu}$

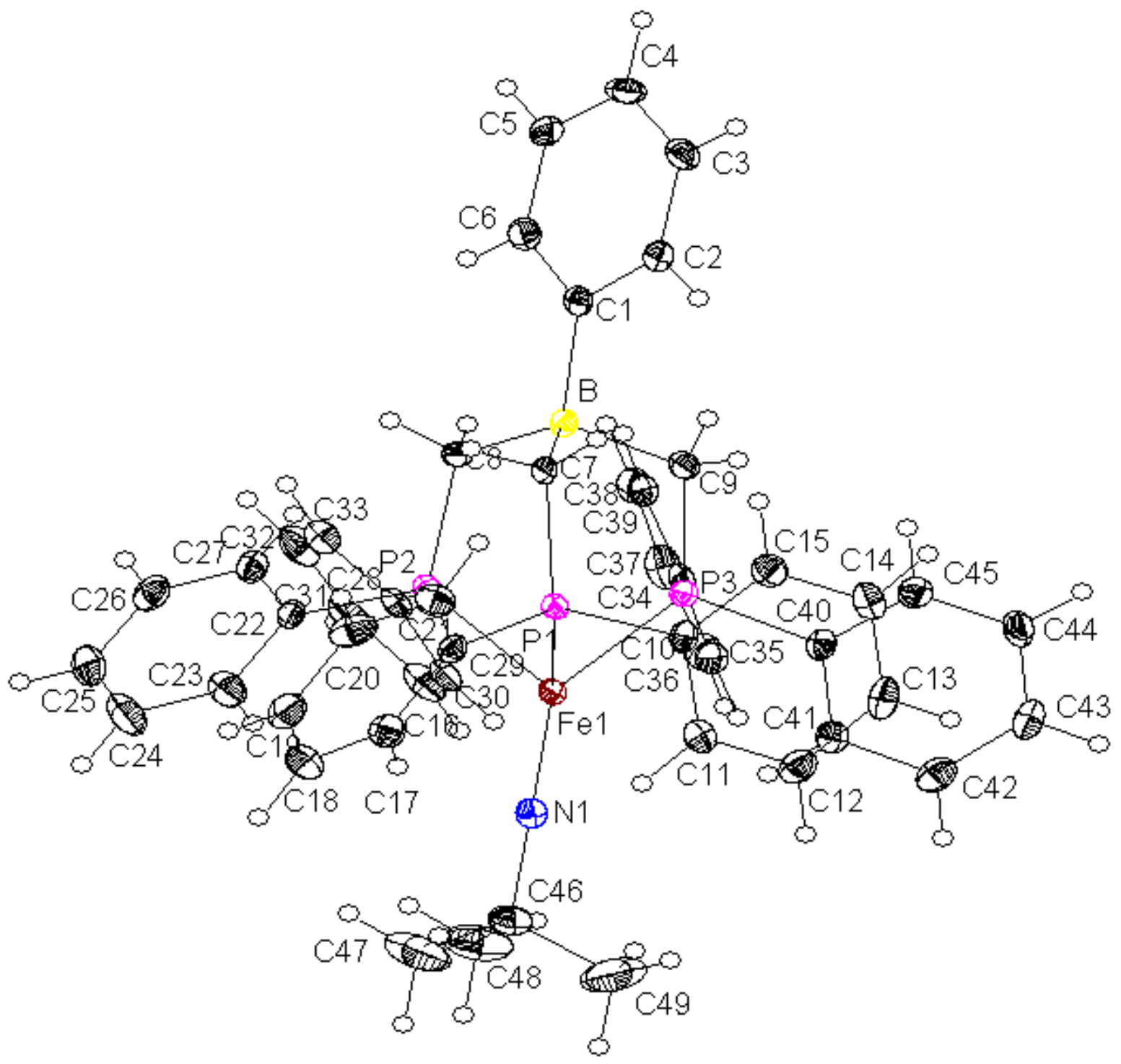


Table S2. Atomic coordinates ( $\mathrm{x} 10^{4}$ ) and equivalent isotropic displacement parameters $\left(\AA^{2} \times 10^{3}\right)$ for $\left[\mathrm{PhBP}_{3}\right] \mathrm{Fe}=\mathrm{N}^{t} \mathrm{Bu}$. $\mathrm{U}(\mathrm{eq})$ is defined as the trace of the orthogonalized $\mathrm{U}^{\mathrm{ij}}$ tensor.

\begin{tabular}{|c|c|c|c|c|}
\hline & $\mathrm{x}$ & $\mathrm{y}$ & $\mathrm{z}$ & $\mathrm{U}_{\text {eq }}$ \\
\hline $\mathrm{Fe}(1)$ & 7643(1) & 3055(1) & 6512(1) & $14(1)$ \\
\hline $\mathrm{P}(1)$ & $7256(1)$ & 4136(1) & $6002(1)$ & $13(1)$ \\
\hline $\mathrm{P}(2)$ & 6561(1) & $2598(1)$ & $5533(1)$ & $13(1)$ \\
\hline $\mathrm{P}(3)$ & $8648(1)$ & 2938(1) & $5506(1)$ & $13(1)$ \\
\hline $\mathrm{N}(1)$ & $7695(1)$ & 2796(1) & $7550(1)$ & $16(1)$ \\
\hline B & $7229(1)$ & $3579(1)$ & 4209(1) & $13(1)$ \\
\hline $\mathrm{C}(1)$ & $7135(1)$ & $3769(1)$ & $3151(1)$ & $14(1)$ \\
\hline$C(2)$ & 7593(1) & 4353(1) & 2848(1) & $16(1)$ \\
\hline$C(3)$ & 7614(1) & $4500(1)$ & 1954(1) & 19(1) \\
\hline $\mathrm{C}(4)$ & $7168(1)$ & 4059(1) & 1309(1) & $21(1)$ \\
\hline$C(5)$ & 6704(1) & 3480(1) & 1576(1) & $20(1)$ \\
\hline$C(6)$ & $6689(1)$ & $3343(1)$ & $2477(1)$ & $18(1)$ \\
\hline$C(7)$ & 6946(1) & 4257(1) & 4811(1) & $14(1)$ \\
\hline $\mathrm{C}(8)$ & $6545(1)$ & 2912(1) & $4387(1)$ & $14(1)$ \\
\hline C(9) & 8344(1) & 3411(1) & 4474(1) & $15(1)$ \\
\hline $\mathrm{C}(10)$ & $8239(1)$ & 4710(1) & $6348(1)$ & $14(1)$ \\
\hline $\mathrm{C}(11)$ & 8482(1) & 4817(1) & $7258(1)$ & $18(1)$ \\
\hline$C(12)$ & 9242(1) & $5209(1)$ & $7569(1)$ & $21(1)$ \\
\hline$C(13)$ & 9793(1) & $5488(1)$ & 6975(1) & $22(1)$ \\
\hline$C(14)$ & 9563(1) & 5384(1) & 6073(1) & $23(1)$ \\
\hline$C(15)$ & 8783(1) & $5005(1)$ & $5760(1)$ & $19(1)$ \\
\hline$C(16)$ & 6344(1) & 4536(1) & 6553(1) & $14(1)$ \\
\hline $\mathrm{C}(17)$ & $6000(1)$ & $4185(1)$ & 7243(1) & $20(1)$ \\
\hline $\mathrm{C}(18)$ & $5274(1)$ & 4462(1) & $7640(1)$ & $25(1)$ \\
\hline $\mathrm{C}(19)$ & 4890(1) & 5092(1) & 7347(1) & $25(1)$ \\
\hline$C(20)$ & $5238(1)$ & 5451(1) & $6669(1)$ & $25(1)$ \\
\hline $\mathrm{C}(21)$ & 5958(1) & $5176(1)$ & $6270(1)$ & $21(1)$ \\
\hline$C(22)$ & $5420(1)$ & $2725(1)$ & 5881(1) & $15(1)$ \\
\hline$C(23)$ & 5113(1) & $2256(1)$ & 6485(1) & $22(1)$ \\
\hline$C(24)$ & $4278(1)$ & $2348(1)$ & $6794(1)$ & $30(1)$ \\
\hline$C(25)$ & 3741(1) & 2922(1) & $6522(1)$ & $26(1)$ \\
\hline$C(26)$ & 4039(1) & $3402(1)$ & $5936(1)$ & 21(1) \\
\hline$C(27)$ & $4875(1)$ & 3301(1) & $5612(1)$ & $17(1)$ \\
\hline$C(28)$ & 6577(1) & $1635(1)$ & $5440(1)$ & $15(1)$ \\
\hline$C(29)$ & 7202(1) & $1232(1)$ & $5978(1)$ & $18(1)$ \\
\hline $\mathrm{C}(30)$ & 7224(1) & $509(1)$ & $5872(1)$ & $25(1)$ \\
\hline $\mathrm{C}(31)$ & 6611(1) & 182(1) & $5252(1)$ & $28(1)$ \\
\hline$C(32)$ & $5968(1)$ & $575(1)$ & $4723(1)$ & $26(1)$ \\
\hline$C(33)$ & 5954(1) & $1299(1)$ & $4812(1)$ & $21(1)$ \\
\hline$C(34)$ & $8848(1)$ & 2042(1) & $5158(1)$ & $14(1)$ \\
\hline$C(35)$ & $9334(1)$ & $1580(1)$ & $5751(1)$ & $18(1)$ \\
\hline$C(36)$ & 9439(1) & $883(1)$ & 5524(1) & $23(1)$ \\
\hline$C(37)$ & $9050(1)$ & 633(1) & $4708(1)$ & $23(1)$ \\
\hline$C(38)$ & $8572(1)$ & 1086(1) & $4107(1)$ & $21(1)$ \\
\hline $\mathrm{C}(39)$ & 8474(1) & 1785(1) & $4325(1)$ & $17(1)$ \\
\hline $\mathrm{C}(40)$ & 9799(1) & $3224(1)$ & $5969(1)$ & $15(1)$ \\
\hline $\mathrm{C}(41)$ & 9961(1) & $3444(1)$ & $6848(1)$ & $18(1)$ \\
\hline
\end{tabular}




\begin{tabular}{lrrrr}
$\mathrm{C}(42)$ & $10815(1)$ & $3709(1)$ & $7186(1)$ & $22(1)$ \\
$\mathrm{C}(43)$ & $11505(1)$ & $3748(1)$ & $6651(1)$ & $20(1)$ \\
$\mathrm{C}(44)$ & $11351(1)$ & $3517(1)$ & $5774(1)$ & $22(1)$ \\
$\mathrm{C}(45)$ & $10505(1)$ & $3256(1)$ & $5438(1)$ & $20(1)$ \\
$\mathrm{C}(46)$ & $7726(1)$ & $2569(1)$ & $8465(1)$ & $27(1)$ \\
$\mathrm{C}(47)$ & $6907(1)$ & $2882(1)$ & $8882(1)$ & $51(1)$ \\
$\mathrm{C}(48)$ & $7693(1)$ & $1785(1)$ & $8493(1)$ & $46(1)$ \\
$\mathrm{C}(49)$ & $8602(1)$ & $2866(1)$ & $8967(1)$ & $52(1)$ \\
\hline
\end{tabular}


Table S3. Bond lengths $[\AA]$ and angles $\left[{ }^{\circ}\right]$ for $\left[\mathrm{PhBP}_{3}\right] \mathrm{Fe} \equiv \mathrm{N}^{t} \mathrm{Bu}$

\begin{tabular}{|c|c|c|c|}
\hline $\mathrm{Fe}(1)-\mathrm{N}(1)$ & $1.6346(13)$ & $\mathrm{C}(19)-\mathrm{C}(20)$ & $1.379(2)$ \\
\hline $\mathrm{Fe}(1)-\mathrm{P}(2)$ & $2.2153(5)$ & $\mathrm{C}(19)-\mathrm{H}(19)$ & 0.95 \\
\hline $\mathrm{Fe}(1)-\mathrm{P}(1)$ & $2.2427(5)$ & $\mathrm{C}(20)-\mathrm{C}(21)$ & $1.382(2)$ \\
\hline $\mathrm{Fe}(1)-\mathrm{P}(3)$ & $2.2531(5)$ & $\mathrm{C}(20)-\mathrm{H}(20)$ & 0.95 \\
\hline $\mathrm{N}(1)-\mathrm{C}(46)$ & $1.442(2)$ & $\mathrm{C}(21)-\mathrm{H}(21)$ & 0.95 \\
\hline $\mathrm{P}(1)-\mathrm{C}(7)$ & $1.8154(16)$ & $\mathrm{C}(22)-\mathrm{C}(27)$ & $1.388(2)$ \\
\hline $\mathrm{P}(1)-\mathrm{C}(16)$ & $1.8271(16)$ & $\mathrm{C}(22)-\mathrm{C}(23)$ & $1.389(2)$ \\
\hline$P(1)-C(10)$ & $1.8358(16)$ & $\mathrm{C}(23)-\mathrm{C}(24)$ & $1.376(2)$ \\
\hline $\mathrm{P}(2)-\mathrm{C}(8)$ & $1.8272(16)$ & $\mathrm{C}(23)-\mathrm{H}(23)$ & 0.95 \\
\hline$P(2)-C(22)$ & $1.8343(16)$ & $\mathrm{C}(24)-\mathrm{C}(25)$ & $1.380(2)$ \\
\hline $\mathrm{P}(2)-\mathrm{C}(28)$ & $1.8358(16)$ & $\mathrm{C}(24)-\mathrm{H}(24)$ & 0.95 \\
\hline $\mathrm{P}(3)-\mathrm{C}(9)$ & $1.8073(16)$ & $\mathrm{C}(25)-\mathrm{C}(26)$ & $1.378(2)$ \\
\hline$P(3)-C(34)$ & $1.8177(16)$ & $\mathrm{C}(25)-\mathrm{H}(25)$ & 0.95 \\
\hline $\mathrm{P}(3)-\mathrm{C}(40)$ & $1.8343(16)$ & $\mathrm{C}(26)-\mathrm{C}(27)$ & $1.391(2)$ \\
\hline $\mathrm{B}-\mathrm{C}(1)$ & $1.627(2)$ & $\mathrm{C}(26)-\mathrm{H}(26)$ & 0.95 \\
\hline B-C(8) & $1.659(2)$ & $\mathrm{C}(27)-\mathrm{H}(27)$ & 0.95 \\
\hline B-C(7) & $1.659(2)$ & $\mathrm{C}(28)-\mathrm{C}(29)$ & $1.383(2)$ \\
\hline B-C(9) & $1.672(2)$ & $\mathrm{C}(28)-\mathrm{C}(33)$ & $1.392(2)$ \\
\hline$C(1)-C(6)$ & $1.399(2)$ & $\mathrm{C}(29)-\mathrm{C}(30)$ & $1.384(2)$ \\
\hline$C(1)-C(2)$ & $1.404(2)$ & $\mathrm{C}(29)-\mathrm{H}(29)$ & 0.95 \\
\hline$C(2)-C(3)$ & $1.381(2)$ & $C(30)-C(31)$ & $1.368(3)$ \\
\hline $\mathrm{C}(2)-\mathrm{H}(2)$ & 0.95 & $\mathrm{C}(30)-\mathrm{H}(30)$ & 0.95 \\
\hline$C(3)-C(4)$ & $1.388(2)$ & $\mathrm{C}(31)-\mathrm{C}(32)$ & $1.382(3)$ \\
\hline $\mathrm{C}(3)-\mathrm{H}(3)$ & 0.95 & $\mathrm{C}(31)-\mathrm{H}(31)$ & 0.95 \\
\hline$C(4)-C(5)$ & $1.380(2)$ & $\mathrm{C}(32)-\mathrm{C}(33)$ & $1.382(2)$ \\
\hline $\mathrm{C}(4)-\mathrm{H}(4)$ & 0.95 & $\mathrm{C}(32)-\mathrm{H}(32)$ & 0.95 \\
\hline$C(5)-C(6)$ & $1.388(2)$ & $\mathrm{C}(33)-\mathrm{H}(33)$ & 0.95 \\
\hline $\mathrm{C}(5)-\mathrm{H}(5)$ & 0.95 & $\mathrm{C}(34)-\mathrm{C}(35)$ & $1.388(2)$ \\
\hline $\mathrm{C}(6)-\mathrm{H}(6)$ & 0.95 & $\mathrm{C}(34)-\mathrm{C}(39)$ & $1.397(2)$ \\
\hline $\mathrm{C}(7)-\mathrm{H}(7 \mathrm{~A})$ & 0.99 & $\mathrm{C}(35)-\mathrm{C}(36)$ & $1.383(2)$ \\
\hline $\mathrm{C}(7)-\mathrm{H}(7 \mathrm{~B})$ & 0.99 & $\mathrm{C}(35)-\mathrm{H}(35)$ & 0.95 \\
\hline $\mathrm{C}(8)-\mathrm{H}(8 \mathrm{~A})$ & 0.99 & $\mathrm{C}(36)-\mathrm{C}(37)$ & $1.378(2)$ \\
\hline $\mathrm{C}(8)-\mathrm{H}(8 \mathrm{~B})$ & 0.99 & $\mathrm{C}(36)-\mathrm{H}(36)$ & 0.95 \\
\hline $\mathrm{C}(9)-\mathrm{H}(9 \mathrm{~A})$ & 0.99 & $\mathrm{C}(37)-\mathrm{C}(38)$ & $1.380(2)$ \\
\hline C(9)-H(9B) & 0.99 & $\mathrm{C}(37)-\mathrm{H}(37)$ & 0.95 \\
\hline$C(10)-C(15)$ & $1.384(2)$ & $\mathrm{C}(38)-\mathrm{C}(39)$ & $1.381(2)$ \\
\hline$C(10)-C(11)$ & $1.391(2)$ & $\mathrm{C}(38)-\mathrm{H}(38)$ & 0.95 \\
\hline$C(11)-C(12)$ & $1.379(2)$ & $\mathrm{C}(39)-\mathrm{H}(39)$ & 0.95 \\
\hline $\mathrm{C}(11)-\mathrm{H}(11)$ & 0.95 & $\mathrm{C}(40)-\mathrm{C}(41)$ & $1.383(2)$ \\
\hline$C(12)-C(13)$ & $1.385(2)$ & $\mathrm{C}(40)-\mathrm{C}(45)$ & $1.388(2)$ \\
\hline $\mathrm{C}(12)-\mathrm{H}(12)$ & 0.95 & $\mathrm{C}(41)-\mathrm{C}(42)$ & $1.393(2)$ \\
\hline$C(13)-C(14)$ & $1.376(2)$ & $\mathrm{C}(41)-\mathrm{H}(41)$ & 0.95 \\
\hline $\mathrm{C}(13)-\mathrm{H}(13)$ & 0.95 & $\mathrm{C}(42)-\mathrm{C}(43)$ & $1.374(2)$ \\
\hline$C(14)-C(15)$ & $1.388(2)$ & $\mathrm{C}(42)-\mathrm{H}(42)$ & 0.95 \\
\hline $\mathrm{C}(14)-\mathrm{H}(14)$ & 0.95 & $\mathrm{C}(43)-\mathrm{C}(44)$ & $1.386(2)$ \\
\hline $\mathrm{C}(15)-\mathrm{H}(15)$ & 0.95 & $\mathrm{C}(43)-\mathrm{H}(43)$ & 0.95 \\
\hline$C(16)-C(17)$ & $1.382(2)$ & $\mathrm{C}(44)-\mathrm{C}(45)$ & $1.379(2)$ \\
\hline$C(16)-C(21)$ & $1.388(2)$ & $\mathrm{C}(44)-\mathrm{H}(44)$ & 0.95 \\
\hline $\mathrm{C}(17)-\mathrm{C}(18)$ & $1.389(2)$ & $\mathrm{C}(45)-\mathrm{H}(45)$ & 0.95 \\
\hline $\mathrm{C}(17)-\mathrm{H}(17)$ & 0.95 & $\mathrm{C}(46)-\mathrm{C}(48)$ & $1.492(3)$ \\
\hline $\mathrm{C}(18)-\mathrm{C}(19)$ & $1.374(2)$ & $C(46)-C(49)$ & $1.523(3)$ \\
\hline $\mathrm{C}(18)-\mathrm{H}(18)$ & 0.95 & $\mathrm{C}(46)-\mathrm{C}(47)$ & $1.546(3)$ \\
\hline
\end{tabular}




\begin{tabular}{|c|c|c|c|}
\hline $\mathrm{C}(47)-\mathrm{H}(47 \mathrm{~A})$ & 0.98 & $\mathrm{C}(4)-\mathrm{C}(5)-\mathrm{H}(5)$ & 119.9 \\
\hline $\mathrm{C}(47)-\mathrm{H}(47 \mathrm{~B})$ & 0.98 & $\mathrm{C}(6)-\mathrm{C}(5)-\mathrm{H}(5)$ & 119.9 \\
\hline $\mathrm{C}(47)-\mathrm{H}(47 \mathrm{C})$ & 0.98 & $C(5)-C(6)-C(1)$ & $122.73(16)$ \\
\hline $\mathrm{C}(48)-\mathrm{H}(48 \mathrm{~A})$ & 0.98 & $\mathrm{C}(5)-\mathrm{C}(6)-\mathrm{H}(6)$ & 118.6 \\
\hline C(48)-H(48B) & 0.98 & $\mathrm{C}(1)-\mathrm{C}(6)-\mathrm{H}(6)$ & 118.6 \\
\hline $\mathrm{C}(48)-\mathrm{H}(48 \mathrm{C})$ & 0.98 & $\mathrm{~B}-\mathrm{C}(7)-\mathrm{P}(1)$ & $113.18(11)$ \\
\hline C(49)-H(49A) & 0.98 & $\mathrm{~B}-\mathrm{C}(7)-\mathrm{H}(7 \mathrm{~A})$ & 108.9 \\
\hline C(49)-H(49B) & 0.98 & $\mathrm{P}(1)-\mathrm{C}(7)-\mathrm{H}(7 \mathrm{~A})$ & 108.9 \\
\hline \multirow[t]{2}{*}{ C(49)-H(49C) } & 0.98 & B-C(7)-H(7B) & 108.9 \\
\hline & & $\mathrm{P}(1)-\mathrm{C}(7)-\mathrm{H}(7 \mathrm{~B})$ & 108.9 \\
\hline $\mathrm{N}(1)-\mathrm{Fe}(1)-\mathrm{P}(2)$ & $117.83(5)$ & $\mathrm{H}(7 \mathrm{~A})-\mathrm{C}(7)-\mathrm{H}(7 \mathrm{~B})$ & 107.8 \\
\hline $\mathrm{N}(1)-\mathrm{Fe}(1)-\mathrm{P}(1)$ & $125.80(5)$ & $\mathrm{B}-\mathrm{C}(8)-\mathrm{P}(2)$ & $117.66(11)$ \\
\hline $\mathrm{P}(2)-\mathrm{Fe}(1)-\mathrm{P}(1)$ & 89.961(18) & $\mathrm{B}-\mathrm{C}(8)-\mathrm{H}(8 \mathrm{~A})$ & 107.9 \\
\hline $\mathrm{N}(1)-\mathrm{Fe}(1)-\mathrm{P}(3)$ & $131.04(5)$ & $\mathrm{P}(2)-\mathrm{C}(8)-\mathrm{H}(8 \mathrm{~A})$ & 107.9 \\
\hline $\mathrm{P}(2)-\mathrm{Fe}(1)-\mathrm{P}(3)$ & 89.381(18) & B-C(8)-H(8B) & 107.9 \\
\hline $\mathrm{P}(1)-\mathrm{Fe}(1)-\mathrm{P}(3)$ & $91.157(17)$ & $\mathrm{P}(2)-\mathrm{C}(8)-\mathrm{H}(8 \mathrm{~B})$ & 107.9 \\
\hline $\mathrm{C}(46)-\mathrm{N}(1)-\mathrm{Fe}(1)$ & 179.17(13) & $\mathrm{H}(8 \mathrm{~A})-\mathrm{C}(8)-\mathrm{H}(8 \mathrm{~B})$ & 107.2 \\
\hline C(7)-P(1)-C(16) & $106.68(7)$ & B-C(9)-P(3) & 115.71(11) \\
\hline C(7)-P(1)-C(10) & 107.49(7) & $\mathrm{B}-\mathrm{C}(9)-\mathrm{H}(9 \mathrm{~A})$ & 108.4 \\
\hline $\mathrm{C}(16)-\mathrm{P}(1)-\mathrm{C}(10)$ & $102.72(7)$ & $\mathrm{P}(3)-\mathrm{C}(9)-\mathrm{H}(9 \mathrm{~A})$ & 108.4 \\
\hline C(7)-P(1)-Fe(1) & $118.59(5)$ & B-C(9)-H(9B) & 108.4 \\
\hline $\mathrm{C}(16)-\mathrm{P}(1)-\mathrm{Fe}(1)$ & $113.28(5)$ & $\mathrm{P}(3)-\mathrm{C}(9)-\mathrm{H}(9 \mathrm{~B})$ & 108.4 \\
\hline $\mathrm{C}(10)-\mathrm{P}(1)-\mathrm{Fe}(1)$ & $106.79(5)$ & H(9A)-C(9)-H(9B) & 107.4 \\
\hline C(8)-P(2)-C(22) & 108.67(7) & $\mathrm{C}(15)-\mathrm{C}(10)-\mathrm{C}(11)$ & $118.29(15)$ \\
\hline $\mathrm{C}(8)-\mathrm{P}(2)-\mathrm{C}(28)$ & $104.68(7)$ & C(15)-C(10)-P(1) & $123.80(13)$ \\
\hline $\mathrm{C}(22)-\mathrm{P}(2)-\mathrm{C}(28)$ & $100.03(7)$ & C(11)-C(10)-P(1) & $117.77(12)$ \\
\hline C(8)-P(2)-Fe(1) & $115.35(5)$ & $C(12)-C(11)-C(10)$ & $121.04(16)$ \\
\hline $\mathrm{C}(22)-\mathrm{P}(2)-\mathrm{Fe}(1)$ & $111.46(5)$ & $\mathrm{C}(12)-\mathrm{C}(11)-\mathrm{H}(11)$ & 119.5 \\
\hline $\mathrm{C}(28)-\mathrm{P}(2)-\mathrm{Fe}(1)$ & $115.34(6)$ & $\mathrm{C}(10)-\mathrm{C}(11)-\mathrm{H}(11)$ & 119.5 \\
\hline C(9)-P(3)-C(34) & $104.40(7)$ & $\mathrm{C}(11)-\mathrm{C}(12)-\mathrm{C}(13)$ & $120.09(17)$ \\
\hline C(9)-P(3)-C(40) & $107.49(7)$ & $\mathrm{C}(11)-\mathrm{C}(12)-\mathrm{H}(12)$ & 120 \\
\hline C(34)-P(3)-C(40) & $102.34(7)$ & $\mathrm{C}(13)-\mathrm{C}(12)-\mathrm{H}(12)$ & 120 \\
\hline C(9)-P(3)-Fe(1) & $114.64(5)$ & $C(14)-C(13)-C(12)$ & $119.42(17)$ \\
\hline $\mathrm{C}(34)-\mathrm{P}(3)-\mathrm{Fe}(1)$ & $115.50(5)$ & $\mathrm{C}(14)-\mathrm{C}(13)-\mathrm{H}(13)$ & 120.3 \\
\hline $\mathrm{C}(40)-\mathrm{P}(3)-\mathrm{Fe}(1)$ & $111.40(6)$ & $\mathrm{C}(12)-\mathrm{C}(13)-\mathrm{H}(15)$ & $120.38(17)$ \\
\hline $\mathrm{C}(1)-\mathrm{B}-\mathrm{C}(8)$ & $110.29(13)$ & $\mathrm{C}(13)-\mathrm{C}(14)-\mathrm{H}(14)$ & 119.8 \\
\hline $\mathrm{C}(1)-\mathrm{B}-\mathrm{C}(7)$ & $111.53(13)$ & $\mathrm{C}(15)-\mathrm{C}(14)-\mathrm{H}(14)$ & 119.8 \\
\hline C(8)-B-C(7) & $107.98(13)$ & $C(10)-C(15)-C(14)$ & $120.73(16)$ \\
\hline $\mathrm{C}(1)-\mathrm{B}-\mathrm{C}(9)$ & $103.98(13)$ & $\mathrm{C}(10)-\mathrm{C}(15)-\mathrm{H}(15)$ & 119.6 \\
\hline C(8)-B-C(9) & $114.37(13)$ & $\mathrm{C}(14)-\mathrm{C}(15)-\mathrm{H}(15)$ & 119.6 \\
\hline C(7)-B-C(9) & 108.71(13) & $C(17)-C(16)-C(21)$ & $118.73(15)$ \\
\hline$C(6)-C(1)-C(2)$ & $115.04(15)$ & C(17)-C(16)-P(1) & $119.85(12)$ \\
\hline$C(6)-C(1)-B$ & $124.09(14)$ & $\mathrm{C}(21)-\mathrm{C}(16)-\mathrm{P}(1)$ & $121.36(13)$ \\
\hline C(2)-C(1)-B & $120.54(14)$ & $C(16)-C(17)-C(18)$ & $120.83(16)$ \\
\hline$C(3)-C(2)-C(1)$ & $123.12(15)$ & $\mathrm{C}(16)-\mathrm{C}(17)-\mathrm{H}(17)$ & 119.6 \\
\hline $\mathrm{C}(3)-\mathrm{C}(2)-\mathrm{H}(2)$ & 118.4 & $\mathrm{C}(18)-\mathrm{C}(17)-\mathrm{H}(17)$ & 119.6 \\
\hline $\mathrm{C}(1)-\mathrm{C}(2)-\mathrm{H}(2)$ & 118.4 & $C(19)-C(18)-C(17)$ & $120.00(17)$ \\
\hline$C(2)-C(3)-C(4)$ & 119.81(16) & $\mathrm{C}(19)-\mathrm{C}(18)-\mathrm{H}(18)$ & 120 \\
\hline $\mathrm{C}(2)-\mathrm{C}(3)-\mathrm{H}(3)$ & 120.1 & $\mathrm{C}(17)-\mathrm{C}(18)-\mathrm{H}(18)$ & 120 \\
\hline $\mathrm{C}(4)-\mathrm{C}(3)-\mathrm{H}(3)$ & 120.1 & $C(18)-C(19)-C(20)$ & $119.55(17)$ \\
\hline$C(5)-C(4)-C(3)$ & $119.09(16)$ & $\mathrm{C}(18)-\mathrm{C}(19)-\mathrm{H}(19)$ & 120.2 \\
\hline $\mathrm{C}(5)-\mathrm{C}(4)-\mathrm{H}(4)$ & 120.5 & $\mathrm{C}(20)-\mathrm{C}(19)-\mathrm{H}(19)$ & 120.2 \\
\hline $\mathrm{C}(3)-\mathrm{C}(4)-\mathrm{H}(4)$ & 120.5 & $\mathrm{C}(19)-\mathrm{C}(20)-\mathrm{C}(21)$ & $120.65(17)$ \\
\hline$C(4)-C(5)-C(6)$ & $120.21(16)$ & $\mathrm{C}(19)-\mathrm{C}(20)-\mathrm{H}(20)$ & 119.7 \\
\hline
\end{tabular}




\begin{tabular}{|c|c|c|c|}
\hline $\mathrm{C}(21)-\mathrm{C}(20)-\mathrm{H}(20)$ & 119.7 & $\mathrm{C}(36)-\mathrm{C}(37)-\mathrm{H}(37)$ & 120.1 \\
\hline$C(20)-C(21)-C(16)$ & $120.24(17)$ & $\mathrm{C}(38)-\mathrm{C}(37)-\mathrm{H}(37)$ & 120.1 \\
\hline $\mathrm{C}(20)-\mathrm{C}(21)-\mathrm{H}(21)$ & 119.9 & $\mathrm{C}(37)-\mathrm{C}(38)-\mathrm{C}(39)$ & $120.22(16)$ \\
\hline $\mathrm{C}(16)-\mathrm{C}(21)-\mathrm{H}(21)$ & 119.9 & $\mathrm{C}(37)-\mathrm{C}(38)-\mathrm{H}(38)$ & 119.9 \\
\hline$C(27)-C(22)-C(23)$ & $118.39(15)$ & $\mathrm{C}(39)-\mathrm{C}(38)-\mathrm{H}(38)$ & 119.9 \\
\hline C(27)-C(22)-P(2) & $122.49(13)$ & $\mathrm{C}(38)-\mathrm{C}(39)-\mathrm{C}(34)$ & $120.63(16)$ \\
\hline $\mathrm{C}(23)-\mathrm{C}(22)-\mathrm{P}(2)$ & $118.96(12)$ & $\mathrm{C}(38)-\mathrm{C}(39)-\mathrm{H}(39)$ & 119.7 \\
\hline$C(24)-C(23)-C(22)$ & $120.96(16)$ & $\mathrm{C}(34)-\mathrm{C}(39)-\mathrm{H}(39)$ & 119.7 \\
\hline $\mathrm{C}(24)-\mathrm{C}(23)-\mathrm{H}(23)$ & 119.5 & $C(41)-C(40)-C(45)$ & $119.05(15)$ \\
\hline $\mathrm{C}(22)-\mathrm{C}(23)-\mathrm{H}(23)$ & 119.5 & C(41)-C(40)-P(3) & $119.92(12)$ \\
\hline$C(23)-C(24)-C(25)$ & $120.17(17)$ & $\mathrm{C}(45)-\mathrm{C}(40)-\mathrm{P}(3)$ & $120.96(13)$ \\
\hline $\mathrm{C}(23)-\mathrm{C}(24)-\mathrm{H}(24)$ & 119.9 & $C(40)-C(41)-C(42)$ & $120.29(16)$ \\
\hline $\mathrm{C}(25)-\mathrm{C}(24)-\mathrm{H}(24)$ & 119.9 & $\mathrm{C}(40)-\mathrm{C}(41)-\mathrm{H}(41)$ & 119.9 \\
\hline$C(26)-C(25)-C(24)$ & 119.91(16) & $\mathrm{C}(42)-\mathrm{C}(41)-\mathrm{H}(41)$ & 119.9 \\
\hline $\mathrm{C}(26)-\mathrm{C}(25)-\mathrm{H}(25)$ & 120 & $\mathrm{C}(43)-\mathrm{C}(42)-\mathrm{C}(41)$ & $120.15(16)$ \\
\hline $\mathrm{C}(24)-\mathrm{C}(25)-\mathrm{H}(25)$ & 120 & $\mathrm{C}(43)-\mathrm{C}(42)-\mathrm{H}(42)$ & 119.9 \\
\hline$C(25)-C(26)-C(27)$ & $119.81(16)$ & $\mathrm{C}(41)-\mathrm{C}(43)-\mathrm{C}(44)$ & $119.78(16)$ \\
\hline $\mathrm{C}(25)-\mathrm{C}(26)-\mathrm{H}(26)$ & 120.1 & $\mathrm{C}(42)-\mathrm{C}(43)-\mathrm{H}(43)$ & 120.1 \\
\hline $\mathrm{C}(27)-\mathrm{C}(26)-\mathrm{H}(26)$ & 120.1 & $\mathrm{C}(44)-\mathrm{C}(43)-\mathrm{H}(43)$ & 120.1 \\
\hline$C(22)-C(27)-C(26)$ & $120.73(16)$ & $C(45)-C(44)-C(43)$ & $120.08(16)$ \\
\hline $\mathrm{C}(22)-\mathrm{C}(27)-\mathrm{H}(27)$ & 119.6 & $\mathrm{C}(45)-\mathrm{C}(44)-\mathrm{H}(44)$ & 120 \\
\hline $\mathrm{C}(26)-\mathrm{C}(27)-\mathrm{H}(27)$ & 119.6 & $\mathrm{C}(43)-\mathrm{C}(44)-\mathrm{H}(44)$ & 120 \\
\hline$C(29)-C(28)-C(33)$ & $118.80(15)$ & $C(44)-C(45)-C(40)$ & $120.62(16)$ \\
\hline C(29)-C(28)-P(2) & $121.44(13)$ & $\mathrm{C}(44)-\mathrm{C}(45)-\mathrm{H}(45)$ & 119.7 \\
\hline C(33)-C(28)-P(2) & $119.76(13)$ & $\mathrm{C}(40)-\mathrm{C}(45)-\mathrm{H}(45)$ & 119.7 \\
\hline$C(28)-C(29)-C(30)$ & $120.37(17)$ & $\mathrm{N}(1)-\mathrm{C}(46)-\mathrm{C}(48)$ & $109.19(15)$ \\
\hline $\mathrm{C}(28)-\mathrm{C}(29)-\mathrm{H}(29)$ & 119.8 & N(1)-C(46)-C(49) & $107.03(15)$ \\
\hline $\mathrm{C}(30)-\mathrm{C}(29)-\mathrm{H}(29)$ & 119.8 & $\mathrm{C}(48)-\mathrm{C}(46)-\mathrm{C}(49)$ & $112.68(17)$ \\
\hline$C(31)-C(30)-C(29)$ & $120.51(17)$ & N(1)-C(46)-C(47) & $109.78(15)$ \\
\hline $\mathrm{C}(31)-\mathrm{C}(30)-\mathrm{H}(30)$ & 119.7 & $\mathrm{C}(48)-\mathrm{C}(46)-\mathrm{C}(47)$ & $110.09(17)$ \\
\hline $\mathrm{C}(29)-\mathrm{C}(30)-\mathrm{H}(30)$ & 119.7 & $\mathrm{C}(49)-\mathrm{C}(46)-\mathrm{C}(47)$ & $108.00(17)$ \\
\hline $\mathrm{C}(30)-\mathrm{C}(31)-\mathrm{C}(32)$ & $119.85(17)$ & $\mathrm{C}(46)-\mathrm{C}(47)-\mathrm{H}(47 \mathrm{~A})$ & 109.5 \\
\hline $\mathrm{C}(30)-\mathrm{C}(31)-\mathrm{H}(31)$ & 120.1 & $\mathrm{C}(46)-\mathrm{C}(47)-\mathrm{H}(47 \mathrm{~B})$ & 109.5 \\
\hline $\mathrm{C}(32)-\mathrm{C}(31)-\mathrm{H}(31)$ & 120.1 & $\mathrm{H}(47 \mathrm{~A})-\mathrm{C}(47)-\mathrm{H}(47 \mathrm{~B})$ & 109.5 \\
\hline$C(31)-C(32)-C(33)$ & $120.00(17)$ & $\mathrm{C}(46)-\mathrm{C}(47)-\mathrm{H}(47 \mathrm{C})$ & 109.5 \\
\hline $\mathrm{C}(31)-\mathrm{C}(32)-\mathrm{H}(32)$ & 120 & $\mathrm{H}(47 \mathrm{~A})-\mathrm{C}(47)-\mathrm{H}(47 \mathrm{C})$ & 109.5 \\
\hline $\mathrm{C}(33)-\mathrm{C}(32)-\mathrm{H}(32)$ & 120 & $\mathrm{H}(47 \mathrm{~B})-\mathrm{C}(47)-\mathrm{H}(47 \mathrm{C})$ & 109.5 \\
\hline $\mathrm{C}(32)-\mathrm{C}(33)-\mathrm{C}(28)$ & $120.44(17)$ & $\mathrm{C}(46)-\mathrm{C}(48)-\mathrm{H}(48 \mathrm{~A})$ & 109.5 \\
\hline $\mathrm{C}(32)-\mathrm{C}(33)-\mathrm{H}(33)$ & 119.8 & $\mathrm{C}(46)-\mathrm{C}(48)-\mathrm{H}(48 \mathrm{~B})$ & 109.5 \\
\hline $\mathrm{C}(28)-\mathrm{C}(33)-\mathrm{H}(33)$ & 119.8 & $\mathrm{H}(48 \mathrm{~A})-\mathrm{C}(48)-\mathrm{H}(48 \mathrm{~B})$ & 109.5 \\
\hline $\mathrm{C}(35)-\mathrm{C}(34)-\mathrm{C}(39)$ & $118.35(15)$ & $\mathrm{C}(46)-\mathrm{C}(48)-\mathrm{H}(48 \mathrm{C})$ & 109.5 \\
\hline C(35)-C(34)-P(3) & $119.65(12)$ & $\mathrm{H}(48 \mathrm{~A})-\mathrm{C}(48)-\mathrm{H}(48 \mathrm{C})$ & 109.5 \\
\hline $\mathrm{C}(39)-\mathrm{C}(34)-\mathrm{P}(3)$ & $121.86(12)$ & $\mathrm{H}(48 \mathrm{~B})-\mathrm{C}(48)-\mathrm{H}(48 \mathrm{C})$ & 109.5 \\
\hline $\mathrm{C}(36)-\mathrm{C}(35)-\mathrm{C}(34)$ & $120.69(16)$ & $\mathrm{C}(46)-\mathrm{C}(49)-\mathrm{H}(49 \mathrm{~A})$ & 109.5 \\
\hline $\mathrm{C}(36)-\mathrm{C}(35)-\mathrm{H}(35)$ & 119.7 & $\mathrm{C}(46)-\mathrm{C}(49)-\mathrm{H}(49 \mathrm{~B})$ & 109.5 \\
\hline $\mathrm{C}(34)-\mathrm{C}(35)-\mathrm{H}(35)$ & 119.7 & $\mathrm{H}(49 \mathrm{~A})-\mathrm{C}(49)-\mathrm{H}(49 \mathrm{~B})$ & 109.5 \\
\hline $\mathrm{C}(37)-\mathrm{C}(36)-\mathrm{C}(35)$ & $120.38(16)$ & $\mathrm{C}(46)-\mathrm{C}(49)-\mathrm{H}(49 \mathrm{C})$ & 109.5 \\
\hline $\mathrm{C}(37)-\mathrm{C}(36)-\mathrm{H}(36)$ & 119.8 & $\mathrm{H}(49 \mathrm{~A})-\mathrm{C}(49)-\mathrm{H}(49 \mathrm{C})$ & 109.5 \\
\hline $\mathrm{C}(35)-\mathrm{C}(36)-\mathrm{H}(36)$ & 119.8 & $\mathrm{H}(49 \mathrm{~B})-\mathrm{C}(49)-\mathrm{H}(49 \mathrm{C})$ & 109.5 \\
\hline $\mathrm{C}(36)-\mathrm{C}(37)-\mathrm{C}(38)$ & 119.71(16) & & \\
\hline
\end{tabular}

Symmetry transformations used to generate equivalent atoms:

$\# 1-\mathrm{x}+1,-\mathrm{y}+2,-\mathrm{z}$ 
Table S4. Anisotropic displacement parameters $\left(\AA^{2} \times 10^{4}\right)$ for $\left[\mathrm{PhBP}_{3}\right] \mathrm{Fe} \equiv \mathrm{N}^{t} \mathrm{Bu}$. The anisotropic displacement factor exponent takes the form: $-2 \pi^{2}\left[\mathrm{~h}^{2} \mathrm{a}^{* 2} \mathrm{U}{ }^{11}+\ldots+2 \mathrm{~h} \mathrm{k} \mathrm{a}\right.$ $\left.\mathrm{b}^{*} \mathrm{U}^{12}\right]$

\begin{tabular}{|c|c|c|c|c|c|c|}
\hline & $\mathrm{U}^{11}$ & $\mathrm{U}^{22}$ & $\mathrm{U}^{33}$ & $\mathrm{U}^{23}$ & $\mathrm{U}^{13}$ & $\mathrm{U}^{12}$ \\
\hline $\mathrm{Fe}(1)$ & 133(1) & 157(1) & 121(1) & $16(1)$ & $25(1)$ & $9(1)$ \\
\hline $\mathrm{N}(1)$ & $149(7)$ & $176(7)$ & $160(7)$ & $8(6)$ & $22(6)$ & $1(6)$ \\
\hline $\mathrm{P}(1)$ & $127(2)$ & $146(2)$ & $128(2)$ & $-5(2)$ & $27(2)$ & $-3(2)$ \\
\hline $\mathrm{P}(2)$ & $125(2)$ & $136(2)$ & $148(2)$ & $18(2)$ & $36(2)$ & $5(2)$ \\
\hline $\mathrm{P}(3)$ & $116(2)$ & $146(2)$ & $127(2)$ & $7(2)$ & $15(2)$ & $11(2)$ \\
\hline B & $130(10)$ & 139(9) & $129(10)$ & $2(7)$ & $4(8)$ & $-13(8)$ \\
\hline $\mathrm{C}(1)$ & 109(8) & $150(8)$ & $155(9)$ & $3(7)$ & $31(7)$ & $35(7)$ \\
\hline$C(2)$ & 141(9) & $165(8)$ & 167(9) & $-3(7)$ & $12(7)$ & 1(7) \\
\hline$C(3)$ & $185(10)$ & 205(9) & 189(9) & $62(7)$ & $51(8)$ & 11(7) \\
\hline$C(4)$ & $238(10)$ & $290(10)$ & 117(9) & $45(8)$ & $35(7)$ & $57(8)$ \\
\hline$C(5)$ & $185(9)$ & $262(10)$ & 143(9) & $-33(8)$ & $2(7)$ & $-8(8)$ \\
\hline$C(6)$ & $169(9)$ & $182(9)$ & 185(9) & $5(7)$ & $27(8)$ & $-7(7)$ \\
\hline$C(7)$ & $112(8)$ & $176(8)$ & $137(8)$ & $26(7)$ & $12(7)$ & $8(7)$ \\
\hline$C(8)$ & $138(8)$ & $168(8)$ & $110(8)$ & $15(7)$ & $14(7)$ & $17(7)$ \\
\hline $\mathrm{C}(9)$ & $154(9)$ & $156(8)$ & 137(9) & $18(7)$ & $35(7)$ & $0(7)$ \\
\hline$C(10)$ & $114(8)$ & $136(8)$ & 181(9) & $-5(7)$ & $12(7)$ & $16(7)$ \\
\hline$C(11)$ & $177(9)$ & 186(9) & 191(9) & $0(7)$ & $52(8)$ & $1(7)$ \\
\hline$C(12)$ & 197(10) & $246(10)$ & $187(10)$ & $-52(8)$ & $-13(8)$ & $22(8)$ \\
\hline$C(13)$ & 143(9) & $205(9)$ & $316(11)$ & $-55(8)$ & $5(8)$ & $-29(7)$ \\
\hline$C(14)$ & 192(10) & $234(10)$ & $265(11)$ & $-2(8)$ & $82(8)$ & $-35(8)$ \\
\hline$C(15)$ & $200(10)$ & 182(9) & 181(9) & $-13(7)$ & $37(8)$ & 2(7) \\
\hline$C(16)$ & $117(8)$ & $170(8)$ & $135(9)$ & $-37(7)$ & $4(7)$ & $2(7)$ \\
\hline$C(17)$ & 191(9) & 195(9) & 197(10) & $7(7)$ & $21(8)$ & $21(8)$ \\
\hline$C(18)$ & 232(10) & $340(11)$ & 198(10) & $-5(8)$ & $88(8)$ & $5(8)$ \\
\hline$C(19)$ & 171(10) & $379(11)$ & 194(10) & $-89(9)$ & $14(8)$ & $74(8)$ \\
\hline$C(20)$ & 280(11) & $272(10)$ & 196(10) & $-36(8)$ & $-26(8)$ & $131(8)$ \\
\hline$C(21)$ & 243(10) & 208(9) & $180(10)$ & $-7(7)$ & $49(8)$ & $15(8)$ \\
\hline$C(22)$ & $112(8)$ & 189(9) & 151(9) & $-28(7)$ & $6(7)$ & $-6(7)$ \\
\hline$C(23)$ & 197(10) & $242(10)$ & $246(10)$ & $76(8)$ & $74(8)$ & $79(8)$ \\
\hline$C(24)$ & 263(11) & $320(11)$ & $331(12)$ & 103(9) & 143(9) & $15(9)$ \\
\hline$C(25)$ & $165(9)$ & $349(11)$ & $282(11)$ & $-16(9)$ & $79(8)$ & $34(8)$ \\
\hline$C(26)$ & 163(9) & 241(10) & $221(10)$ & $-25(8)$ & $-16(8)$ & $61(7)$ \\
\hline$C(27)$ & $150(9)$ & $180(9)$ & $186(9)$ & $-10(7)$ & $-2(7)$ & $-23(7)$ \\
\hline$C(28)$ & $168(9)$ & $141(8)$ & $160(9)$ & $4(7)$ & $89(7)$ & 2(7) \\
\hline$C(29)$ & $175(9)$ & 197(9) & 179(9) & $41(7)$ & $94(8)$ & $7(7)$ \\
\hline$C(30)$ & 283(11) & 208(9) & 284(11) & $97(8)$ & $162(9)$ & $72(8)$ \\
\hline$C(31)$ & 418(13) & $149(9)$ & $323(11)$ & $6(8)$ & 243(10) & $-4(9)$ \\
\hline$C(32)$ & $346(12)$ & 211(10) & $255(11)$ & $-66(8)$ & 140(9) & $-107(9)$ \\
\hline$C(33)$ & 212(10) & 221(10) & $202(10)$ & $20(8)$ & $62(8)$ & $-13(8)$ \\
\hline$C(34)$ & $114(8)$ & $150(8)$ & 170(9) & $16(7)$ & $56(7)$ & $15(7)$ \\
\hline$C(35)$ & 162(9) & 214(9) & 170(9) & $4(7)$ & $24(7)$ & $18(7)$ \\
\hline$C(36)$ & $222(10)$ & $195(9)$ & $273(11)$ & $51(8)$ & $62(8)$ & $80(8)$ \\
\hline$C(37)$ & 209(10) & 166(9) & $343(12)$ & $-44(8)$ & $107(9)$ & $21(8)$ \\
\hline$C(38)$ & 183(10) & $251(10)$ & 199(10) & $-71(8)$ & $56(8)$ & $-5(8)$ \\
\hline $\mathrm{C}(39)$ & $125(9)$ & $217(9)$ & $168(9)$ & $-3(7)$ & $34(7)$ & $15(7)$ \\
\hline $\mathrm{C}(40)$ & $122(8)$ & $129(8)$ & $183(9)$ & $19(7)$ & $3(7)$ & $22(7)$ \\
\hline $\mathrm{C}(41)$ & $159(9)$ & 203(9) & 189(9) & $8(8)$ & $25(7)$ & $13(7)$ \\
\hline
\end{tabular}




$\begin{array}{llllrrr}\mathrm{C}(42) & 225(10) & 219(10) & 201(10) & -53(8) & -30(8) & 12(8) \\ \mathrm{C}(43) & 139(9) & 153(9) & 294(11) & 4(8) & -23(8) & -5(7) \\ \mathrm{C}(44) & 146(9) & 275(10) & 262(10) & 16(8) & 73(8) & 13(8) \\ \mathrm{C}(45) & 183(9) & 244(9) & 159(9) & -13(8) & 20(7) & 17(7) \\ \mathrm{C}(46) & 303(11) & 309(11) & 186(10) & 108(8) & 27(9) & 12(9) \\ \mathrm{C}(47) & 657(17) & 642(16) & 287(13) & 167(11) & 228(12) & 194(13) \\ \mathrm{C}(48) & 791(18) & 333(12) & 262(12) & 126(9) & 73(12) & 8(11) \\ \mathrm{C}(49) & 686(17) & 573(16) & 248(12) & 77(11) & -162(12) & -121(13)\end{array}$


Table S5. Hydrogen coordinates ( $\left.\mathrm{x} 10^{4}\right)$ and isotropic displacement parameters $\left(\AA^{2} \mathrm{x} 10\right.$ 3) for $\left[\mathrm{PhBP}_{3}\right] \mathrm{Fe}=\mathrm{N}^{t} \mathrm{Bu}$.

\begin{tabular}{|c|c|c|c|c|}
\hline & $\mathrm{x}$ & $\mathrm{y}$ & $\mathrm{z}$ & $U_{\text {iso }}$ \\
\hline $\mathrm{H}(2)$ & 7904 & 4661 & 3278 & 19 \\
\hline $\mathrm{H}(3)$ & 7932 & 4902 & 1781 & 23 \\
\hline $\mathrm{H}(4)$ & 7182 & 4153 & 693 & 26 \\
\hline $\mathrm{H}(5)$ & 6393 & 3176 & 1141 & 24 \\
\hline $\mathrm{H}(6)$ & 6364 & 2942 & 2644 & 21 \\
\hline $\mathrm{H}(7 \mathrm{~A})$ & 6277 & 4334 & 4691 & 17 \\
\hline $\mathrm{H}(7 \mathrm{~B})$ & 7254 & 4685 & 4619 & 17 \\
\hline $\mathrm{H}(8 \mathrm{~A})$ & 6703 & 2512 & 4015 & 17 \\
\hline $\mathrm{H}(8 \mathrm{~B})$ & 5909 & 3053 & 4168 & 17 \\
\hline $\mathrm{H}(9 \mathrm{~A})$ & 8679 & 3864 & 4511 & 18 \\
\hline $\mathrm{H}(9 \mathrm{~B})$ & 8560 & 3136 & 3983 & 18 \\
\hline $\mathrm{H}(11)$ & 8118 & 4615 & 7671 & 22 \\
\hline $\mathrm{H}(12)$ & 9387 & 5289 & 8191 & 26 \\
\hline $\mathrm{H}(13)$ & 10326 & 5749 & 7188 & 27 \\
\hline $\mathrm{H}(14)$ & 9940 & 5572 & 5663 & 27 \\
\hline $\mathrm{H}(15)$ & 8622 & 4948 & 5135 & 22 \\
\hline $\mathrm{H}(17)$ & 6263 & 3750 & 7448 & 23 \\
\hline $\mathrm{H}(18)$ & 5044 & 4216 & 8113 & 30 \\
\hline $\mathrm{H}(19)$ & 4387 & 5279 & 7611 & 30 \\
\hline $\mathrm{H}(20)$ & 4981 & 5890 & 6474 & 30 \\
\hline $\mathrm{H}(21)$ & 6189 & 5426 & 5800 & 25 \\
\hline $\mathrm{H}(23)$ & 5485 & 1866 & 6689 & 27 \\
\hline $\mathrm{H}(24)$ & 4071 & 2016 & 7195 & 36 \\
\hline $\mathrm{H}(25)$ & 3167 & 2987 & 6739 & 31 \\
\hline $\mathrm{H}(26)$ & 3674 & 3800 & 5753 & 25 \\
\hline $\mathrm{H}(27)$ & 5076 & 3629 & 5203 & 21 \\
\hline H(29) & 7619 & 1452 & 6423 & 21 \\
\hline $\mathrm{H}(30)$ & 7668 & 238 & 6232 & 30 \\
\hline $\mathrm{H}(31)$ & 6628 & -315 & 5185 & 34 \\
\hline $\mathrm{H}(32)$ & 5536 & 349 & 4297 & 31 \\
\hline H(33) & 5516 & 1568 & 4442 & 25 \\
\hline $\mathrm{H}(35)$ & 9598 & 1745 & 6318 & 22 \\
\hline $\mathrm{H}(36)$ & 9780 & 574 & 5932 & 27 \\
\hline $\mathrm{H}(37)$ & 9111 & 151 & 4560 & 28 \\
\hline $\mathrm{H}(38)$ & 8309 & 916 & 3541 & 25 \\
\hline H(39) & 8148 & 2094 & 3905 & 20 \\
\hline $\mathrm{H}(41)$ & 9487 & 3414 & 7222 & 22 \\
\hline $\mathrm{H}(42)$ & 10921 & 3862 & 7789 & 26 \\
\hline $\mathrm{H}(43)$ & 12086 & 3933 & 6880 & 24 \\
\hline $\mathrm{H}(44)$ & 11830 & 3539 & 5404 & 27 \\
\hline $\mathrm{H}(45)$ & 10404 & 3097 & 4837 & 23 \\
\hline $\mathrm{H}(47 \mathrm{~A})$ & 6931 & 3397 & 8855 & 77 \\
\hline $\mathrm{H}(47 \mathrm{~B})$ & 6936 & 2733 & 9507 & 77 \\
\hline $\mathrm{H}(47 \mathrm{C})$ & 6333 & 2716 & 8550 & 77 \\
\hline $\mathrm{H}(48 \mathrm{~A})$ & 7118 & 1621 & 8161 & 69 \\
\hline $\mathrm{H}(48 \mathrm{~B})$ & 7727 & 1628 & 9114 & 69 \\
\hline $\mathrm{H}(48 \mathrm{C})$ & 8211 & 1591 & 8221 & 69 \\
\hline $\mathrm{H}(49 \mathrm{~A})$ & 9133 & 2646 & 8746 & 78 \\
\hline
\end{tabular}


H(49B)

$\mathrm{H}(49 \mathrm{C})$
8615

8622

2768

3376

9606

8873

78

78 
Figure S2. Fully labeled drawing of $\left[\mathrm{PhBP}_{3}\right] \mathrm{Co} \equiv \mathrm{N}^{t} \mathrm{Bu}$

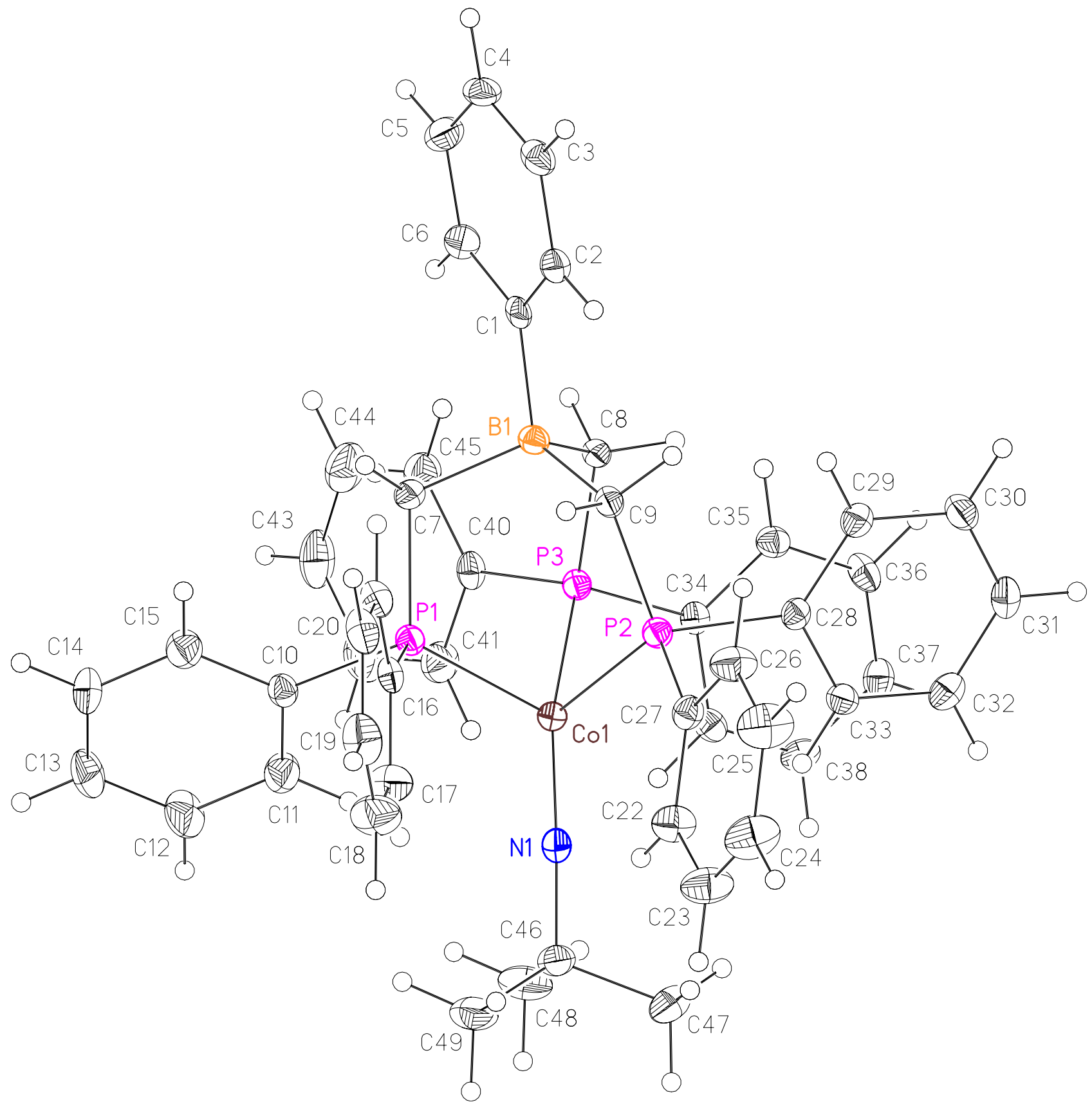


Table S6. Atomic coordinates ( $\mathrm{x} 10^{4}$ ) and equivalent isotropic displacement parameters $\left(\AA^{2} \mathrm{x} 10^{3}\right)$ for $\left[\mathrm{PhBP}_{3}\right] \mathrm{Co} \equiv \mathrm{N}^{t} \mathrm{Bu}$. $\mathrm{U}(\mathrm{eq})$ is defined as the trace of the orthogonalized $\mathrm{U}^{\mathrm{ij}}$ tensor.

\begin{tabular}{|c|c|c|c|c|}
\hline & $\mathrm{x}$ & $\mathrm{y}$ & $\mathrm{z}$ & $\mathrm{U}_{\text {eq }}$ \\
\hline$\overline{\mathrm{Co}(1)}$ & 1044(1) & $310(1)$ & $2316(1)$ & $14(1)$ \\
\hline $\mathrm{P}(1)$ & $1596(1)$ & $1416(1)$ & $2502(1)$ & $14(1)$ \\
\hline $\mathrm{P}(2)$ & 2511(1) & $137(1)$ & 1841(1) & $14(1)$ \\
\hline $\mathrm{P}(3)$ & $-105(1)$ & $618(1)$ & $1518(1)$ & $14(1)$ \\
\hline $\mathrm{N}(1)$ & $690(1)$ & $-233(1)$ & $2805(1)$ & $16(1)$ \\
\hline $\mathrm{B}(1)$ & 1872(2) & $1527(1)$ & $1306(1)$ & $15(1)$ \\
\hline$C(1)$ & $2243(2)$ & $2125(1)$ & $860(1)$ & $15(1)$ \\
\hline$C(2)$ & $3382(2)$ & $2167(1)$ & $709(1)$ & $18(1)$ \\
\hline$C(3)$ & $3691(2)$ & $2697(1)$ & $342(1)$ & $22(1)$ \\
\hline$C(4)$ & 2861(2) & $3212(1)$ & $115(1)$ & $23(1)$ \\
\hline$C(5)$ & $1726(2)$ & $3192(1)$ & $254(1)$ & $24(1)$ \\
\hline$C(6)$ & 1432(2) & 2661(1) & 619(1) & $20(1)$ \\
\hline$C(7)$ & $1573(2)$ & 1981(1) & $1878(1)$ & $15(1)$ \\
\hline$C(8)$ & $653(2)$ & 1061(1) & 1001(1) & $14(1)$ \\
\hline C(9) & $2980(2)$ & $935(1)$ & 1495(1) & $15(1)$ \\
\hline$C(10)$ & $737(2)$ & 1872(1) & 2987(1) & $16(1)$ \\
\hline$C(11)$ & $-135(2)$ & $1510(1)$ & $3220(1)$ & $26(1)$ \\
\hline$C(12)$ & $-734(2)$ & 1852(1) & 3611(1) & $33(1)$ \\
\hline$C(13)$ & $-458(2)$ & 2556(1) & 3772(1) & $29(1)$ \\
\hline$C(14)$ & $406(2)$ & 2925(1) & 3541(1) & $27(1)$ \\
\hline$C(15)$ & $993(2)$ & 2589(1) & $3148(1)$ & $24(1)$ \\
\hline$C(16)$ & $3117(2)$ & 1476(1) & 2923(1) & $16(1)$ \\
\hline$C(17)$ & $3373(2)$ & 1071(1) & 3424(1) & $26(1)$ \\
\hline$C(18)$ & $4505(2)$ & 1091(1) & 3761(1) & $33(1)$ \\
\hline$C(19)$ & $5404(2)$ & 1510(1) & 3603(1) & $29(1)$ \\
\hline$C(20)$ & $5160(2)$ & 1914(1) & 3107(1) & $25(1)$ \\
\hline$C(21)$ & $4030(2)$ & 1896(1) & 2769(1) & $20(1)$ \\
\hline$C(22)$ & 3773(2) & $-619(1)$ & 2783(1) & $25(1)$ \\
\hline$C(23)$ & 4754(2) & $-985(1)$ & 3082(1) & $31(1)$ \\
\hline$C(24)$ & $5829(2)$ & $-987(1)$ & 2886(1) & $35(1)$ \\
\hline$C(25)$ & $5925(2)$ & $-623(1)$ & 2386(1) & $36(1)$ \\
\hline$C(26)$ & 4943(2) & $-264(1)$ & 2082(1) & $27(1)$ \\
\hline$C(27)$ & $3850(2)$ & $-263(1)$ & $2275(1)$ & $17(1)$ \\
\hline$C(28)$ & $2174(2)$ & $-570(1)$ & $1289(1)$ & $15(1)$ \\
\hline$C(29)$ & 2491(2) & $-525(1)$ & $747(1)$ & $18(1)$ \\
\hline$C(30)$ & $2207(2)$ & $-1085(1)$ & $353(1)$ & $21(1)$ \\
\hline$C(31)$ & 1613(2) & $-1690(1)$ & 499(1) & $22(1)$ \\
\hline$C(32)$ & $1305(2)$ & $-1747(1)$ & $1038(1)$ & $21(1)$ \\
\hline$C(33)$ & $1578(2)$ & $-1189(1)$ & $1428(1)$ & $17(1)$ \\
\hline$C(34)$ & $-850(2)$ & $-203(1)$ & 1194(1) & $14(1)$ \\
\hline$C(35)$ & $-1003(2)$ & $-346(1)$ & $609(1)$ & $17(1)$ \\
\hline$C(36)$ & $-1504(2)$ & $-992(1)$ & $392(1)$ & $21(1)$ \\
\hline$C(37)$ & $-1873(2)$ & $-1503(1)$ & $748(1)$ & $20(1)$ \\
\hline$C(38)$ & $-1755(2)$ & $-1362(1)$ & $1330(1)$ & $20(1)$ \\
\hline$C(39)$ & $-1232(2)$ & $-721(1)$ & 1551(1) & $18(1)$ \\
\hline $\mathrm{C}(40)$ & $-1406(2)$ & $1182(1)$ & 1599(1) & $17(1)$ \\
\hline
\end{tabular}




\begin{tabular}{lrrrr}
$\mathrm{C}(41)$ & $-2125(2)$ & $957(1)$ & $1986(1)$ & $26(1)$ \\
$\mathrm{C}(42)$ & $-3150(2)$ & $1337(1)$ & $2050(1)$ & $34(1)$ \\
$\mathrm{C}(43)$ & $-3461(2)$ & $1954(1)$ & $1735(1)$ & $33(1)$ \\
$\mathrm{C}(44)$ & $-2758(2)$ & $2193(1)$ & $1355(1)$ & $34(1)$ \\
$\mathrm{C}(45)$ & $-1736(2)$ & $1808(1)$ & $1283(1)$ & $24(1)$ \\
$\mathrm{C}(46)$ & $429(2)$ & $-691(1)$ & $3260(1)$ & $20(1)$ \\
$\mathrm{C}(47)$ & $829(2)$ & $-1463(1)$ & $3154(1)$ & $32(1)$ \\
$\mathrm{C}(48)$ & $-928(2)$ & $-683(1)$ & $3262(1)$ & $32(1)$ \\
$\mathrm{C}(49)$ & $1106(2)$ & $-405(1)$ & $3834(1)$ & $31(1)$ \\
$\mathrm{C}(51)$ & $3373(3)$ & $5392(2)$ & $9674(1)$ & $63(1)$ \\
$\mathrm{C}(52)$ & $3748(3)$ & $5989(2)$ & $10031(1)$ & $59(1)$ \\
$\mathrm{C}(53)$ & $2923(3)$ & $6460(1)$ & $10176(1)$ & $61(1)$ \\
$\mathrm{C}(54)$ & $1706(2)$ & $6332(1)$ & $9944(1)$ & $63(1)$ \\
$\mathrm{C}(55)$ & $1363(3)$ & $5735(2)$ & $9621(1)$ & $66(1)$ \\
$\mathrm{C}(56)$ & $2183(3)$ & $5271(2)$ & $9457(1)$ & $63(1)$ \\
$\mathrm{C}(61)$ & $5313(2)$ & $9279(1)$ & $73(1)$ & $28(1)$ \\
$\mathrm{C}(62)$ & $5745(2)$ & $9778(1)$ & $495(1)$ & $27(1)$ \\
$\mathrm{C}(63)$ & $5431(2)$ & $10498(1)$ & $424(1)$ & $26(1)$ \\
& & & & \\
\hline
\end{tabular}


Table S7. Bond lengths $[\AA]$ and angles $\left[{ }^{\circ}\right]$ for $\left[\mathrm{PhBP}_{3}\right] \mathrm{Co} \equiv \mathrm{N}^{t} \mathrm{Bu}$

\begin{tabular}{|c|c|c|c|}
\hline $\mathrm{Co}(1)-\mathrm{N}(1)$ & $1.6326(15)$ & $C(19)-C(20)$ & $1.380(3)$ \\
\hline $\mathrm{Co}(1)-\mathrm{P}(1)$ & $2.1595(5)$ & C(19)-H(19) & 0.9500 \\
\hline $\operatorname{Co}(1)-\mathrm{P}(3)$ & $2.1765(5)$ & $C(20)-C(21)$ & $1.384(3)$ \\
\hline $\operatorname{Co}(1)-P(2)$ & $2.1823(5)$ & $\mathrm{C}(20)-\mathrm{H}(20)$ & 0.9500 \\
\hline $\mathrm{P}(1)-\mathrm{C}(7)$ & $1.8057(17)$ & $\mathrm{C}(21)-\mathrm{H}(21)$ & 0.9500 \\
\hline $\mathrm{P}(1)-\mathrm{C}(10)$ & $1.8311(18)$ & $\mathrm{C}(22)-\mathrm{C}(23)$ & $1.384(3)$ \\
\hline $\mathrm{P}(1)-\mathrm{C}(16)$ & $1.8344(19)$ & $\mathrm{C}(22)-\mathrm{C}(27)$ & $1.387(2)$ \\
\hline $\mathrm{P}(2)-\mathrm{C}(9)$ & $1.8087(17)$ & $\mathrm{C}(22)-\mathrm{H}(22)$ & 0.9500 \\
\hline $\mathrm{P}(2)-\mathrm{C}(27)$ & $1.8304(18)$ & $\mathrm{C}(23)-\mathrm{C}(24)$ & $1.372(3)$ \\
\hline $\mathrm{P}(2)-\mathrm{C}(28)$ & $1.8408(18)$ & $\mathrm{C}(23)-\mathrm{H}(23)$ & 0.9500 \\
\hline $\mathrm{P}(3)-\mathrm{C}(8)$ & $1.8062(17)$ & $\mathrm{C}(24)-\mathrm{C}(25)$ & $1.384(3)$ \\
\hline$P(3)-C(34)$ & $1.8361(18)$ & $\mathrm{C}(24)-\mathrm{H}(24)$ & 0.9500 \\
\hline $\mathrm{P}(3)-\mathrm{C}(40)$ & $1.8377(18)$ & $C(25)-C(26)$ & $1.383(3)$ \\
\hline N(1)-C(46) & $1.441(2)$ & $\mathrm{C}(25)-\mathrm{H}(25)$ & 0.9500 \\
\hline B(1)-C(1) & $1.633(3)$ & $\mathrm{C}(26)-\mathrm{C}(27)$ & $1.389(2)$ \\
\hline $\mathrm{B}(1)-\mathrm{C}(9)$ & $1.664(3)$ & $\mathrm{C}(26)-\mathrm{H}(26)$ & 0.9500 \\
\hline $\mathrm{B}(1)-\mathrm{C}(8)$ & $1.677(3)$ & $\mathrm{C}(28)-\mathrm{C}(33)$ & $1.394(2)$ \\
\hline $\mathrm{B}(1)-\mathrm{C}(7)$ & $1.678(3)$ & $\mathrm{C}(28)-\mathrm{C}(29)$ & $1.395(2)$ \\
\hline$C(1)-C(2)$ & $1.396(2)$ & $C(29)-C(30)$ & $1.394(2)$ \\
\hline$C(1)-C(6)$ & $1.402(2)$ & C(29)-H(29) & 0.9500 \\
\hline$C(2)-C(3)$ & $1.394(2)$ & $\mathrm{C}(30)-\mathrm{C}(31)$ & $1.379(2)$ \\
\hline $\mathrm{C}(2)-\mathrm{H}(2)$ & 0.9500 & $\mathrm{C}(30)-\mathrm{H}(30)$ & 0.9500 \\
\hline$C(3)-C(4)$ & $1.377(3)$ & $\mathrm{C}(31)-\mathrm{C}(32)$ & $1.384(2)$ \\
\hline $\mathrm{C}(3)-\mathrm{H}(3)$ & 0.9500 & $\mathrm{C}(31)-\mathrm{H}(31)$ & 0.9500 \\
\hline$C(4)-C(5)$ & $1.378(3)$ & $C(32)-C(33)$ & $1.384(2)$ \\
\hline $\mathrm{C}(4)-\mathrm{H}(4)$ & 0.9500 & $\mathrm{C}(32)-\mathrm{H}(32)$ & 0.9500 \\
\hline$C(5)-C(6)$ & $1.384(2)$ & $\mathrm{C}(33)-\mathrm{H}(33)$ & 0.9500 \\
\hline $\mathrm{C}(5)-\mathrm{H}(5)$ & 0.9500 & $C(34)-C(35)$ & $1.392(2)$ \\
\hline $\mathrm{C}(6)-\mathrm{H}(6)$ & 0.9500 & $C(34)-C(39)$ & $1.394(2)$ \\
\hline $\mathrm{C}(7)-\mathrm{H}(7 \mathrm{~A})$ & 0.9900 & $C(35)-C(36)$ & $1.382(2)$ \\
\hline C(7)-H(7B) & 0.9900 & $\mathrm{C}(35)-\mathrm{H}(35)$ & 0.9500 \\
\hline $\mathrm{C}(8)-\mathrm{H}(8 \mathrm{~A})$ & 0.9900 & $C(36)-C(37)$ & $1.379(2)$ \\
\hline $\mathrm{C}(8)-\mathrm{H}(8 \mathrm{~B})$ & 0.9900 & $\mathrm{C}(36)-\mathrm{H}(36)$ & 0.9500 \\
\hline $\mathrm{C}(9)-\mathrm{H}(9 \mathrm{~A})$ & 0.9900 & $\mathrm{C}(37)-\mathrm{C}(38)$ & $1.386(2)$ \\
\hline C(9)-H(9B) & 0.9900 & $\mathrm{C}(37)-\mathrm{H}(37)$ & 0.9500 \\
\hline $\mathrm{C}(10)-\mathrm{C}(11)$ & $1.383(2)$ & $\mathrm{C}(38)-\mathrm{C}(39)$ & $1.384(2)$ \\
\hline $\mathrm{C}(10)-\mathrm{C}(15)$ & $1.394(2)$ & $\mathrm{C}(38)-\mathrm{H}(38)$ & 0.9500 \\
\hline $\mathrm{C}(11)-\mathrm{C}(12)$ & $1.391(3)$ & C(39)-H(39) & 0.9500 \\
\hline $\mathrm{C}(11)-\mathrm{H}(11)$ & 0.9500 & $C(40)-C(41)$ & $1.389(2)$ \\
\hline$C(12)-C(13)$ & $1.376(3)$ & $C(40)-C(45)$ & $1.394(2)$ \\
\hline $\mathrm{C}(12)-\mathrm{H}(12)$ & 0.9500 & $C(41)-C(42)$ & $1.384(3)$ \\
\hline $\mathrm{C}(13)-\mathrm{C}(14)$ & $1.380(3)$ & $\mathrm{C}(41)-\mathrm{H}(41)$ & 0.9500 \\
\hline $\mathrm{C}(13)-\mathrm{H}(13)$ & 0.9500 & $\mathrm{C}(42)-\mathrm{C}(43)$ & $1.374(3)$ \\
\hline $\mathrm{C}(14)-\mathrm{C}(15)$ & $1.380(3)$ & $\mathrm{C}(42)-\mathrm{H}(42)$ & 0.9500 \\
\hline $\mathrm{C}(14)-\mathrm{H}(14)$ & 0.9500 & $C(43)-C(44)$ & $1.373(3)$ \\
\hline $\mathrm{C}(15)-\mathrm{H}(15)$ & 0.9500 & $\mathrm{C}(43)-\mathrm{H}(43)$ & 0.9500 \\
\hline$C(16)-C(21)$ & $1.389(2)$ & $C(44)-C(45)$ & $1.391(3)$ \\
\hline$C(16)-C(17)$ & $1.390(3)$ & $\mathrm{C}(44)-\mathrm{H}(44)$ & 0.9500 \\
\hline $\mathrm{C}(17)-\mathrm{C}(18)$ & $1.385(3)$ & $\mathrm{C}(45)-\mathrm{H}(45)$ & 0.9500 \\
\hline C(17)-H(17) & 0.9500 & $C(46)-C(47)$ & $1.529(3)$ \\
\hline C(18)-C(19) & $1.378(3)$ & $C(46)-C(49)$ & $1.533(3)$ \\
\hline $\mathrm{C}(18)-\mathrm{H}(18)$ & 0.9500 & $C(46)-C(48)$ & $1.533(3)$ \\
\hline
\end{tabular}




\begin{tabular}{|c|c|c|c|}
\hline $\mathrm{C}(47)-\mathrm{H}(47 \mathrm{~A})$ & 0.9800 & $\mathrm{C}(1)-\mathrm{B}(1)-\mathrm{C}(9)$ & $110.73(15)$ \\
\hline C(47)-H(47B) & 0.9800 & $\mathrm{C}(1)-\mathrm{B}(1)-\mathrm{C}(8)$ & $110.60(14)$ \\
\hline $\mathrm{C}(47)-\mathrm{H}(47 \mathrm{C})$ & 0.9800 & $\mathrm{C}(9)-\mathrm{B}(1)-\mathrm{C}(8)$ & $107.55(14)$ \\
\hline $\mathrm{C}(48)-\mathrm{H}(48 \mathrm{~A})$ & 0.9800 & $\mathrm{C}(1)-\mathrm{B}(1)-\mathrm{C}(7)$ & $107.14(14)$ \\
\hline $\mathrm{C}(48)-\mathrm{H}(48 \mathrm{~B})$ & 0.9800 & $\mathrm{C}(9)-\mathrm{B}(1)-\mathrm{C}(7)$ & $110.99(15)$ \\
\hline $\mathrm{C}(48)-\mathrm{H}(48 \mathrm{C})$ & 0.9800 & $\mathrm{C}(8)-\mathrm{B}(1)-\mathrm{C}(7)$ & $109.85(14)$ \\
\hline C(49)-H(49A) & 0.9800 & $C(2)-C(1)-C(6)$ & $114.92(16)$ \\
\hline C(49)-H(49B) & 0.9800 & $\mathrm{C}(2)-\mathrm{C}(1)-\mathrm{B}(1)$ & $123.71(16)$ \\
\hline $\mathrm{C}(49)-\mathrm{H}(49 \mathrm{C})$ & 0.9800 & $\mathrm{C}(6)-\mathrm{C}(1)-\mathrm{B}(1)$ & $121.31(16)$ \\
\hline$C(51)-C(56)$ & $1.372(4)$ & $\mathrm{C}(3)-\mathrm{C}(2)-\mathrm{C}(1)$ & $122.79(18)$ \\
\hline$C(51)-C(52)$ & $1.410(4)$ & $\mathrm{C}(3)-\mathrm{C}(2)-\mathrm{H}(2)$ & 118.6 \\
\hline $\mathrm{C}(51)-\mathrm{H}(51)$ & 0.9500 & $\mathrm{C}(1)-\mathrm{C}(2)-\mathrm{H}(2)$ & 118.6 \\
\hline$C(52)-C(53)$ & $1.363(3)$ & $C(4)-C(3)-C(2)$ & $120.02(18)$ \\
\hline $\mathrm{C}(52)-\mathrm{H}(52)$ & 0.9500 & $\mathrm{C}(4)-\mathrm{C}(3)-\mathrm{H}(3)$ & 120.0 \\
\hline $\mathrm{C}(53)-\mathrm{C}(54)$ & $1.408(4)$ & $\mathrm{C}(2)-\mathrm{C}(3)-\mathrm{H}(3)$ & 120.0 \\
\hline $\mathrm{C}(53)-\mathrm{H}(53)$ & 0.9500 & $C(3)-C(4)-C(5)$ & $119.17(18)$ \\
\hline$C(54)-C(55)$ & $1.361(3)$ & $\mathrm{C}(3)-\mathrm{C}(4)-\mathrm{H}(4)$ & 120.4 \\
\hline $\mathrm{C}(54)-\mathrm{H}(54)$ & 0.9500 & $\mathrm{C}(5)-\mathrm{C}(4)-\mathrm{H}(4)$ & 120.4 \\
\hline$C(55)-C(56)$ & $1.366(4)$ & $C(4)-C(5)-C(6)$ & $120.07(19)$ \\
\hline $\mathrm{C}(55)-\mathrm{H}(55)$ & 0.9500 & $\mathrm{C}(4)-\mathrm{C}(5)-\mathrm{H}(5)$ & 120.0 \\
\hline $\mathrm{C}(56)-\mathrm{H}(56)$ & 0.9500 & $\mathrm{C}(6)-\mathrm{C}(5)-\mathrm{H}(5)$ & 120.0 \\
\hline $\mathrm{C}(61)-\mathrm{C}(62)$ & $1.385(3)$ & $C(5)-C(6)-C(1)$ & $123.02(18)$ \\
\hline C(61)-C(63)\#1 & $1.385(3)$ & $\mathrm{C}(5)-\mathrm{C}(6)-\mathrm{H}(6)$ & 118.5 \\
\hline $\mathrm{C}(61)-\mathrm{H}(61)$ & 0.9500 & $\mathrm{C}(1)-\mathrm{C}(6)-\mathrm{H}(6)$ & 118.5 \\
\hline $\mathrm{C}(62)-\mathrm{C}(63)$ & $1.378(3)$ & $\mathrm{B}(1)-\mathrm{C}(7)-\mathrm{P}(1)$ & $113.38(12)$ \\
\hline $\mathrm{C}(62)-\mathrm{H}(62)$ & 0.9500 & $\mathrm{~B}(1)-\mathrm{C}(7)-\mathrm{H}(7 \mathrm{~A})$ & 108.9 \\
\hline $\mathrm{C}(63)-\mathrm{C}(61) \# 1$ & $1.385(3)$ & $\mathrm{P}(1)-\mathrm{C}(7)-\mathrm{H}(7 \mathrm{~A})$ & 108.9 \\
\hline \multirow[t]{2}{*}{$\mathrm{C}(63)-\mathrm{H}(63)$} & 0.9500 & $\mathrm{~B}(1)-\mathrm{C}(7)-\mathrm{H}(7 \mathrm{~B})$ & 108.9 \\
\hline & & $\mathrm{P}(1)-\mathrm{C}(7)-\mathrm{H}(7 \mathrm{~B})$ & 108.9 \\
\hline $\mathrm{N}(1)-\mathrm{Co}(1)-\mathrm{P}(1)$ & $122.24(5)$ & $\mathrm{H}(7 \mathrm{~A})-\mathrm{C}(7)-\mathrm{H}(7 \mathrm{~B})$ & 107.7 \\
\hline $\mathrm{N}(1)-\mathrm{Co}(1)-\mathrm{P}(3)$ & $126.49(5)$ & $\mathrm{B}(1)-\mathrm{C}(8)-\mathrm{P}(3)$ & $113.01(12)$ \\
\hline $\mathrm{P}(1)-\mathrm{Co}(1)-\mathrm{P}(3)$ & $92.18(2)$ & $\mathrm{B}(1)-\mathrm{C}(8)-\mathrm{H}(8 \mathrm{~A})$ & 109.0 \\
\hline N(1)-Co(1)-P(2) & $125.24(5)$ & $\mathrm{P}(3)-\mathrm{C}(8)-\mathrm{H}(8 \mathrm{~A})$ & 109.0 \\
\hline $\mathrm{P}(1)-\mathrm{Co}(1)-\mathrm{P}(2)$ & $91.46(2)$ & $\mathrm{B}(1)-\mathrm{C}(8)-\mathrm{H}(8 \mathrm{~B})$ & 109.0 \\
\hline $\mathrm{P}(3)-\mathrm{Co}(1)-\mathrm{P}(2)$ & $88.95(2)$ & $\mathrm{P}(3)-\mathrm{C}(8)-\mathrm{H}(8 \mathrm{~B})$ & 109.0 \\
\hline C(7)-P(1)-C(10) & $108.28(8)$ & $\mathrm{H}(8 \mathrm{~A})-\mathrm{C}(8)-\mathrm{H}(8 \mathrm{~B})$ & 107.8 \\
\hline C(7)-P(1)-C(16) & $106.82(9)$ & $\mathrm{B}(1)-\mathrm{C}(9)-\mathrm{P}(2)$ & $113.05(12)$ \\
\hline C(10)-P(1)-C(16) & $100.35(8)$ & $\mathrm{B}(1)-\mathrm{C}(9)-\mathrm{H}(9 \mathrm{~A})$ & 109.0 \\
\hline C(7)-P(1)-Co(1) & 114.61(6) & $\mathrm{P}(2)-\mathrm{C}(9)-\mathrm{H}(9 \mathrm{~A})$ & 109.0 \\
\hline C(10)-P(1)-Co(1) & $113.45(6)$ & $\mathrm{B}(1)-\mathrm{C}(9)-\mathrm{H}(9 \mathrm{~B})$ & 109.0 \\
\hline C(16)-P(1)-Co(1) & $112.21(6)$ & $\mathrm{P}(2)-\mathrm{C}(9)-\mathrm{H}(9 \mathrm{~B})$ & 109.0 \\
\hline C(9)-P(2)-C(27) & $107.76(8)$ & $\mathrm{H}(9 \mathrm{~A})-\mathrm{C}(9)-\mathrm{H}(9 \mathrm{~B})$ & 107.8 \\
\hline $\mathrm{C}(9)-\mathrm{P}(2)-\mathrm{C}(28)$ & $107.24(8)$ & $C(11)-C(10)-C(15)$ & $118.61(17)$ \\
\hline $\mathrm{C}(27)-\mathrm{P}(2)-\mathrm{C}(28)$ & $99.33(8)$ & $\mathrm{C}(11)-\mathrm{C}(10)-\mathrm{P}(1)$ & $121.22(14)$ \\
\hline C(9)-P(2)-Co(1) & $115.02(6)$ & $\mathrm{C}(15)-\mathrm{C}(10)-\mathrm{P}(1)$ & $120.11(14)$ \\
\hline $\mathrm{C}(27)-\mathrm{P}(2)-\mathrm{Co}(1)$ & $113.36(6)$ & $\mathrm{C}(10)-\mathrm{C}(11)-\mathrm{C}(12)$ & $120.56(19)$ \\
\hline $\mathrm{C}(28)-\mathrm{P}(2)-\mathrm{Co}(1)$ & $112.83(6)$ & $\mathrm{C}(10)-\mathrm{C}(11)-\mathrm{H}(11)$ & 119.7 \\
\hline $\mathrm{C}(8)-\mathrm{P}(3)-\mathrm{C}(34)$ & $109.14(8)$ & $\mathrm{C}(12)-\mathrm{C}(11)-\mathrm{H}(11)$ & 119.7 \\
\hline C(8)-P(3)-C(40) & 107.24(9) & $\mathrm{C}(13)-\mathrm{C}(12)-\mathrm{C}(11)$ & $120.09(19)$ \\
\hline $\mathrm{C}(34)-\mathrm{P}(3)-\mathrm{C}(40)$ & $101.23(8)$ & $\mathrm{C}(13)-\mathrm{C}(12)-\mathrm{H}(12)$ & 120.0 \\
\hline $\mathrm{C}(8)-\mathrm{P}(3)-\mathrm{Co}(1)$ & $115.20(6)$ & $\mathrm{C}(11)-\mathrm{C}(12)-\mathrm{H}(12)$ & 120.0 \\
\hline $\mathrm{C}(34)-\mathrm{P}(3)-\mathrm{Co}(1)$ & $107.96(6)$ & $\mathrm{C}(12)-\mathrm{C}(13)-\mathrm{C}(14)$ & 119.94(19) \\
\hline C(40)-P(3)-Co(1) & $115.07(6)$ & $\mathrm{C}(12)-\mathrm{C}(13)-\mathrm{H}(13)$ & 120.0 \\
\hline $\mathrm{C}(46)-\mathrm{N}(1)-\mathrm{Co}(1)$ & $176.68(13)$ & $\mathrm{C}(14)-\mathrm{C}(13)-\mathrm{H}(13)$ & 120.0 \\
\hline
\end{tabular}




\begin{tabular}{|c|c|c|c|}
\hline$C(13)-C(14)-C(15)$ & $120.05(19)$ & $\mathrm{C}(31)-\mathrm{C}(32)-\mathrm{C}(33)$ & $119.83(18)$ \\
\hline $\mathrm{C}(13)-\mathrm{C}(14)-\mathrm{H}(14)$ & 120.0 & $\mathrm{C}(31)-\mathrm{C}(32)-\mathrm{H}(32)$ & 120.1 \\
\hline $\mathrm{C}(15)-\mathrm{C}(14)-\mathrm{H}(14)$ & 120.0 & $\mathrm{C}(33)-\mathrm{C}(32)-\mathrm{H}(32)$ & 120.1 \\
\hline$C(14)-C(15)-C(10)$ & $120.74(18)$ & $C(32)-C(33)-C(28)$ & $120.96(17)$ \\
\hline $\mathrm{C}(14)-\mathrm{C}(15)-\mathrm{H}(15)$ & 119.6 & $\mathrm{C}(32)-\mathrm{C}(33)-\mathrm{H}(33)$ & 119.5 \\
\hline $\mathrm{C}(10)-\mathrm{C}(15)-\mathrm{H}(15)$ & 119.6 & $\mathrm{C}(28)-\mathrm{C}(33)-\mathrm{H}(33)$ & 119.5 \\
\hline$C(21)-C(16)-C(17)$ & $118.08(18)$ & $C(35)-C(34)-C(39)$ & $118.40(16)$ \\
\hline $\mathrm{C}(21)-\mathrm{C}(16)-\mathrm{P}(1)$ & $123.99(14)$ & $\mathrm{C}(35)-\mathrm{C}(34)-\mathrm{P}(3)$ & $122.74(14)$ \\
\hline C(17)-C(16)-P(1) & $117.93(14)$ & C(39)-C(34)-P(3) & $118.79(13)$ \\
\hline$C(18)-C(17)-C(16)$ & $120.79(19)$ & $\mathrm{C}(36)-\mathrm{C}(35)-\mathrm{C}(34)$ & $120.45(17)$ \\
\hline $\mathrm{C}(18)-\mathrm{C}(17)-\mathrm{H}(17)$ & 119.6 & $\mathrm{C}(36)-\mathrm{C}(35)-\mathrm{H}(35)$ & 119.8 \\
\hline $\mathrm{C}(16)-\mathrm{C}(17)-\mathrm{H}(17)$ & 119.6 & $\mathrm{C}(34)-\mathrm{C}(35)-\mathrm{H}(35)$ & 119.8 \\
\hline$C(19)-C(18)-C(17)$ & $120.6(2)$ & $\mathrm{C}(37)-\mathrm{C}(36)-\mathrm{C}(35)$ & $120.69(18)$ \\
\hline $\mathrm{C}(19)-\mathrm{C}(18)-\mathrm{H}(18)$ & 119.7 & $\mathrm{C}(37)-\mathrm{C}(36)-\mathrm{H}(36)$ & 119.7 \\
\hline $\mathrm{C}(17)-\mathrm{C}(18)-\mathrm{H}(18)$ & 119.7 & $\mathrm{C}(35)-\mathrm{C}(36)-\mathrm{H}(36)$ & 119.7 \\
\hline $\mathrm{C}(18)-\mathrm{C}(19)-\mathrm{C}(20)$ & 119.11(19) & $\mathrm{C}(36)-\mathrm{C}(37)-\mathrm{C}(38)$ & $119.60(18)$ \\
\hline $\mathrm{C}(18)-\mathrm{C}(19)-\mathrm{H}(19)$ & 120.4 & $\mathrm{C}(36)-\mathrm{C}(37)-\mathrm{H}(37)$ & 120.2 \\
\hline $\mathrm{C}(20)-\mathrm{C}(19)-\mathrm{H}(19)$ & 120.4 & $\mathrm{C}(38)-\mathrm{C}(37)-\mathrm{H}(37)$ & 120.2 \\
\hline$C(19)-C(20)-C(21)$ & $120.52(19)$ & $\mathrm{C}(39)-\mathrm{C}(38)-\mathrm{C}(37)$ & $119.86(17)$ \\
\hline $\mathrm{C}(19)-\mathrm{C}(20)-\mathrm{H}(20)$ & 119.7 & $\mathrm{C}(39)-\mathrm{C}(38)-\mathrm{H}(38)$ & 120.1 \\
\hline $\mathrm{C}(21)-\mathrm{C}(20)-\mathrm{H}(20)$ & 119.7 & $\mathrm{C}(37)-\mathrm{C}(38)-\mathrm{H}(38)$ & 120.1 \\
\hline$C(20)-C(21)-C(16)$ & $120.89(18)$ & $\mathrm{C}(38)-\mathrm{C}(39)-\mathrm{C}(34)$ & $120.96(17)$ \\
\hline $\mathrm{C}(20)-\mathrm{C}(21)-\mathrm{H}(21)$ & 119.6 & $\mathrm{C}(38)-\mathrm{C}(39)-\mathrm{H}(39)$ & 119.5 \\
\hline $\mathrm{C}(16)-\mathrm{C}(21)-\mathrm{H}(21)$ & 119.6 & $\mathrm{C}(34)-\mathrm{C}(39)-\mathrm{H}(39)$ & 119.5 \\
\hline$C(23)-C(22)-C(27)$ & $120.77(18)$ & $\mathrm{C}(41)-\mathrm{C}(40)-\mathrm{C}(45)$ & $118.06(17)$ \\
\hline $\mathrm{C}(23)-\mathrm{C}(22)-\mathrm{H}(22)$ & 119.6 & C(41)-C(40)-P(3) & $118.03(14)$ \\
\hline $\mathrm{C}(27)-\mathrm{C}(22)-\mathrm{H}(22)$ & 119.6 & C(45)-C(40)-P(3) & $123.88(15)$ \\
\hline$C(24)-C(23)-C(22)$ & $120.30(19)$ & $\mathrm{C}(42)-\mathrm{C}(41)-\mathrm{C}(40)$ & 121.1(2) \\
\hline $\mathrm{C}(24)-\mathrm{C}(23)-\mathrm{H}(23)$ & 119.9 & $\mathrm{C}(42)-\mathrm{C}(41)-\mathrm{H}(41)$ & 119.5 \\
\hline $\mathrm{C}(22)-\mathrm{C}(23)-\mathrm{H}(23)$ & 119.9 & $\mathrm{C}(40)-\mathrm{C}(41)-\mathrm{H}(41)$ & 119.5 \\
\hline$C(23)-C(24)-C(25)$ & $119.6(2)$ & $\mathrm{C}(43)-\mathrm{C}(42)-\mathrm{C}(41)$ & $120.2(2)$ \\
\hline $\mathrm{C}(23)-\mathrm{C}(24)-\mathrm{H}(24)$ & 120.2 & $\mathrm{C}(43)-\mathrm{C}(42)-\mathrm{H}(42)$ & 119.9 \\
\hline $\mathrm{C}(25)-\mathrm{C}(24)-\mathrm{H}(24)$ & 120.2 & $\mathrm{C}(41)-\mathrm{C}(42)-\mathrm{H}(42)$ & 119.9 \\
\hline$C(26)-C(25)-C(24)$ & $120.3(2)$ & $\mathrm{C}(44)-\mathrm{C}(43)-\mathrm{C}(42)$ & $120.0(2)$ \\
\hline $\mathrm{C}(26)-\mathrm{C}(25)-\mathrm{H}(25)$ & 119.9 & $\mathrm{C}(44)-\mathrm{C}(43)-\mathrm{H}(43)$ & 120.0 \\
\hline $\mathrm{C}(24)-\mathrm{C}(25)-\mathrm{H}(25)$ & 119.9 & $\mathrm{C}(42)-\mathrm{C}(43)-\mathrm{H}(43)$ & 120.0 \\
\hline$C(25)-C(26)-C(27)$ & $120.50(18)$ & $\mathrm{C}(43)-\mathrm{C}(44)-\mathrm{C}(45)$ & $120.2(2)$ \\
\hline $\mathrm{C}(25)-\mathrm{C}(26)-\mathrm{H}(26)$ & 119.8 & $\mathrm{C}(43)-\mathrm{C}(44)-\mathrm{H}(44)$ & 119.9 \\
\hline $\mathrm{C}(27)-\mathrm{C}(26)-\mathrm{H}(26)$ & 119.8 & $\mathrm{C}(45)-\mathrm{C}(44)-\mathrm{H}(44)$ & 119.9 \\
\hline$C(22)-C(27)-C(26)$ & $118.54(17)$ & $\mathrm{C}(44)-\mathrm{C}(45)-\mathrm{C}(40)$ & $120.53(19)$ \\
\hline $\mathrm{C}(22)-\mathrm{C}(27)-\mathrm{P}(2)$ & $120.62(14)$ & $\mathrm{C}(44)-\mathrm{C}(45)-\mathrm{H}(45)$ & 119.7 \\
\hline C(26)-C(27)-P(2) & $120.66(14)$ & $\mathrm{C}(40)-\mathrm{C}(45)-\mathrm{H}(45)$ & 119.7 \\
\hline $\mathrm{C}(33)-\mathrm{C}(28)-\mathrm{C}(29)$ & $118.51(16)$ & $\mathrm{N}(1)-\mathrm{C}(46)-\mathrm{C}(47)$ & $108.64(15)$ \\
\hline C(33)-C(28)-P(2) & 117.61(13) & $\mathrm{N}(1)-\mathrm{C}(46)-\mathrm{C}(49)$ & $109.05(15)$ \\
\hline C(29)-C(28)-P(2) & $123.88(14)$ & $\mathrm{C}(47)-\mathrm{C}(46)-\mathrm{C}(49)$ & $110.55(16)$ \\
\hline$C(30)-C(29)-C(28)$ & $120.47(17)$ & $\mathrm{N}(1)-\mathrm{C}(46)-\mathrm{C}(48)$ & $109.26(15)$ \\
\hline $\mathrm{C}(30)-\mathrm{C}(29)-\mathrm{H}(29)$ & 119.8 & $\mathrm{C}(47)-\mathrm{C}(46)-\mathrm{C}(48)$ & $109.46(17)$ \\
\hline $\mathrm{C}(28)-\mathrm{C}(29)-\mathrm{H}(29)$ & 119.8 & C(49)-C(46)-C(48) & $109.86(16)$ \\
\hline $\mathrm{C}(31)-\mathrm{C}(30)-\mathrm{C}(29)$ & $119.97(18)$ & $\mathrm{C}(46)-\mathrm{C}(47)-\mathrm{H}(47 \mathrm{~A})$ & 109.5 \\
\hline $\mathrm{C}(31)-\mathrm{C}(30)-\mathrm{H}(30)$ & 120.0 & $\mathrm{C}(46)-\mathrm{C}(47)-\mathrm{H}(47 \mathrm{~B})$ & 109.5 \\
\hline $\mathrm{C}(29)-\mathrm{C}(30)-\mathrm{H}(30)$ & 120.0 & $\mathrm{H}(47 \mathrm{~A})-\mathrm{C}(47)-\mathrm{H}(47 \mathrm{~B})$ & 109.5 \\
\hline$C(30)-C(31)-C(32)$ & $120.26(18)$ & $\mathrm{C}(46)-\mathrm{C}(47)-\mathrm{H}(47 \mathrm{C})$ & 109.5 \\
\hline $\mathrm{C}(30)-\mathrm{C}(31)-\mathrm{H}(31)$ & 119.9 & $\mathrm{H}(47 \mathrm{~A})-\mathrm{C}(47)-\mathrm{H}(47 \mathrm{C})$ & 109.5 \\
\hline $\mathrm{C}(32)-\mathrm{C}(31)-\mathrm{H}(31)$ & 119.9 & $\mathrm{H}(47 \mathrm{~B})-\mathrm{C}(47)-\mathrm{H}(47 \mathrm{C})$ & 109.5 \\
\hline
\end{tabular}




$\begin{array}{llll}\mathrm{C}(46)-\mathrm{C}(48)-\mathrm{H}(48 \mathrm{~A}) & 109.5 & \mathrm{C}(54)-\mathrm{C}(53)-\mathrm{H}(53) & 121.4 \\ \mathrm{C}(46)-\mathrm{C}(48)-\mathrm{H}(48 \mathrm{~B}) & 109.5 & \mathrm{C}(55)-\mathrm{C}(54)-\mathrm{C}(53) & 121.3(3) \\ \mathrm{H}(48 \mathrm{~A})-\mathrm{C}(48)-\mathrm{H}(48 \mathrm{~B}) & 109.5 & \mathrm{C}(55)-\mathrm{C}(54)-\mathrm{H}(54) & 119.3 \\ \mathrm{C}(46)-\mathrm{C}(48)-\mathrm{H}(48 \mathrm{C}) & 109.5 & \mathrm{C}(53)-\mathrm{C}(54)-\mathrm{H}(54) & 119.3 \\ \mathrm{H}(48 \mathrm{~A})-\mathrm{C}(48)-\mathrm{H}(48 \mathrm{C}) & 109.5 & \mathrm{C}(54)-\mathrm{C}(55)-\mathrm{C}(56) & 121.8(3) \\ \mathrm{H}(48 \mathrm{~B})-\mathrm{C}(48)-\mathrm{H}(48 \mathrm{C}) & 109.5 & \mathrm{C}(54)-\mathrm{C}(55)-\mathrm{H}(55) & 119.1 \\ \mathrm{C}(46)-\mathrm{C}(49)-\mathrm{H}(49 \mathrm{~A}) & 109.5 & \mathrm{C}(56)-\mathrm{C}(55)-\mathrm{H}(55) & 119.1 \\ \mathrm{C}(46)-\mathrm{C}(49)-\mathrm{H}(49 \mathrm{~B}) & 109.5 & \mathrm{C}(55)-\mathrm{C}(56)-\mathrm{C}(51) & 117.3(3) \\ \mathrm{H}(49 \mathrm{~A})-\mathrm{C}(49)-\mathrm{H}(49 \mathrm{~B}) & 109.5 & \mathrm{C}(55)-\mathrm{C}(56)-\mathrm{H}(56) & 121.3 \\ \mathrm{C}(46)-\mathrm{C}(49)-\mathrm{H}(49 \mathrm{C}) & 109.5 & \mathrm{C}(51)-\mathrm{C}(56)-\mathrm{H}(56) & 121.3 \\ \mathrm{H}(49 \mathrm{~A})-\mathrm{C}(49)-\mathrm{H}(49 \mathrm{C}) & 109.5 & \mathrm{C}(62)-\mathrm{C}(61)-\mathrm{C}(63) \# 1 & 120.07(19) \\ \mathrm{H}(49 \mathrm{~B})-\mathrm{C}(49)-\mathrm{H}(49 \mathrm{C}) & 109.5 & \mathrm{C}(62)-\mathrm{C}(61)-\mathrm{H}(61) & 120.0 \\ \mathrm{C}(56)-\mathrm{C}(51)-\mathrm{C}(52) & 121.8(3) & \mathrm{C}(63) \# 1-\mathrm{C}(61)-\mathrm{H}(61) & 120.0 \\ \mathrm{C}(56)-\mathrm{C}(51)-\mathrm{H}(51) & 119.1 & \mathrm{C}(63)-\mathrm{C}(62)-\mathrm{C}(61) & 120.19(19) \\ \mathrm{C}(52)-\mathrm{C}(51)-\mathrm{H}(51) & 119.1 & \mathrm{C}(63)-\mathrm{C}(62)-\mathrm{H}(62) & 119.9 \\ \mathrm{C}(53)-\mathrm{C}(52)-\mathrm{C}(51) & 120.2(3) & \mathrm{C}(61)-\mathrm{C}(62)-\mathrm{H}(62) & 119.9 \\ \mathrm{C}(53)-\mathrm{C}(52)-\mathrm{H}(52) & 119.9 & \mathrm{C}(62)-\mathrm{C}(63)-\mathrm{C}(61) \# 1 & 119.74(19) \\ \mathrm{C}(51)-\mathrm{C}(52)-\mathrm{H}(52) & 119.9 & \mathrm{C}(62)-\mathrm{C}(63)-\mathrm{H}(63) & 120.1 \\ \mathrm{C}(52)-\mathrm{C}(53)-\mathrm{C}(54) & 117.3(3) & \mathrm{C}(61) \# 1-\mathrm{C}(63)-\mathrm{H}(63) & 120.1 \\ \mathrm{C}(52)-\mathrm{C}(53)-\mathrm{H}(53) & 121.4 & & \\ & & & \end{array}$

Symmetry transformations used to generate equivalent atoms:

$\# 1-\mathrm{x}+1,-\mathrm{y}+2,-\mathrm{z}$ 
Table S8. Anisotropic displacement parameters $\left(\AA^{2} \times 10^{4}\right)$ for $\left[\mathrm{PhBP}_{3}\right] \mathrm{Co} \equiv \mathrm{N}^{t} \mathrm{Bu}$. The anisotropic displacement factor exponent takes the form: $-2 \pi^{2}\left[\mathrm{~h}^{2} \mathrm{a}^{* 2} \mathrm{U}{ }^{11}+\ldots+2 \mathrm{~h} \mathrm{k} \mathrm{a}\right.$ $\left.\mathrm{b}^{*} \mathrm{U}^{12}\right]$

\begin{tabular}{|c|c|c|c|c|c|c|}
\hline & $\mathrm{U}^{11}$ & $\mathrm{U}^{22}$ & $\mathrm{U}^{33}$ & $\mathrm{U}^{23}$ & $\mathrm{U}^{13}$ & $\mathrm{U}^{12}$ \\
\hline $\operatorname{Co}(1)$ & $148(1)$ & $133(1)$ & $134(1)$ & $2(1)$ & $30(1)$ & $0(1)$ \\
\hline $\mathrm{P}(1)$ & $152(3)$ & $144(3)$ & $132(2)$ & $-13(2)$ & $28(2)$ & $-7(2)$ \\
\hline $\mathrm{P}(2)$ & $147(3)$ & $147(3)$ & $141(2)$ & $-2(2)$ & $28(2)$ & $3(2)$ \\
\hline $\mathrm{P}(3)$ & $143(3)$ & $138(3)$ & $139(2)$ & $-11(2)$ & $32(2)$ & $-1(2)$ \\
\hline $\mathrm{N}(1)$ & $168(8)$ & $153(8)$ & $165(8)$ & $-23(7)$ & $30(7)$ & $12(7)$ \\
\hline $\mathrm{B}(1)$ & $153(11)$ & $152(11)$ & 131(11) & 2(9) & $30(9)$ & $-19(9)$ \\
\hline $\mathrm{C}(1)$ & $187(10)$ & $149(10)$ & 107(9) & $-54(8)$ & $10(8)$ & $-46(8)$ \\
\hline $\mathrm{C}(2)$ & $224(11)$ & $172(10)$ & $153(10)$ & $-30(8)$ & $25(9)$ & $-21(8)$ \\
\hline $\mathrm{C}(3)$ & $255(12)$ & $228(11)$ & $173(10)$ & $-49(9)$ & $70(9)$ & $-96(9)$ \\
\hline$C(4)$ & $347(13)$ & $192(11)$ & $153(10)$ & $12(9)$ & $49(9)$ & $-108(10)$ \\
\hline$C(5)$ & 291(12) & $182(11)$ & $225(11)$ & $35(9)$ & $2(10)$ & $-8(9)$ \\
\hline$C(6)$ & $205(11)$ & $204(11)$ & 199(11) & $5(9)$ & $47(9)$ & $-5(9)$ \\
\hline $\mathrm{C}(7)$ & $165(10)$ & $139(10)$ & $158(10)$ & $7(8)$ & $31(8)$ & $-10(8)$ \\
\hline $\mathrm{C}(8)$ & $159(10)$ & $146(10)$ & $130(9)$ & $4(8)$ & $39(8)$ & $14(8)$ \\
\hline $\mathrm{C}(9)$ & $156(10)$ & $150(10)$ & $149(10)$ & $-22(8)$ & $47(8)$ & $-23(8)$ \\
\hline$C(10)$ & $160(10)$ & $174(10)$ & $145(10)$ & $-17(8)$ & $9(8)$ & $23(8)$ \\
\hline $\mathrm{C}(11)$ & $301(12)$ & 201(11) & 294(12) & $-30(9)$ & $112(10)$ & $-34(9)$ \\
\hline$C(12)$ & $320(13)$ & $336(13)$ & $389(14)$ & $-66(11)$ & $234(11)$ & $-36(10)$ \\
\hline$C(13)$ & $266(12)$ & $340(13)$ & $282(12)$ & $-88(10)$ & 101(10) & $85(10)$ \\
\hline $\mathrm{C}(14)$ & $324(13)$ & $193(11)$ & $290(12)$ & $-97(9)$ & $62(10)$ & $6(10)$ \\
\hline$C(15)$ & $256(12)$ & $217(11)$ & $250(11)$ & $-24(9)$ & $72(10)$ & $-40(9)$ \\
\hline$C(16)$ & $175(10)$ & $166(10)$ & $153(10)$ & $-61(8)$ & $32(8)$ & $-5(8)$ \\
\hline$C(17)$ & $230(12)$ & $286(12)$ & $247(12)$ & $30(10)$ & $3(10)$ & $-60(10)$ \\
\hline $\mathrm{C}(18)$ & $327(14)$ & $377(14)$ & $246(12)$ & $62(10)$ & $-54(10)$ & $-6(11)$ \\
\hline C(19) & 191(12) & $353(13)$ & $292(12)$ & $-99(10)$ & $-33(10)$ & $15(10)$ \\
\hline $\mathrm{C}(20)$ & $212(12)$ & $265(12)$ & $278(12)$ & $-109(10)$ & $99(10)$ & $-63(9)$ \\
\hline$C(21)$ & $207(11)$ & $217(11)$ & $180(11)$ & $-37(8)$ & $50(9)$ & $-11(9)$ \\
\hline$C(22)$ & $194(11)$ & $290(12)$ & $253(12)$ & 53(9) & $37(9)$ & 21(9) \\
\hline $\mathrm{C}(23)$ & $249(13)$ & $356(14)$ & $308(13)$ & 131(10) & $19(10)$ & $31(10)$ \\
\hline $\mathrm{C}(24)$ & $216(12)$ & $389(14)$ & $410(14)$ & $123(11)$ & $-27(11)$ & $80(10)$ \\
\hline$C(25)$ & $179(12)$ & $448(14)$ & $447(15)$ & $128(12)$ & $78(11)$ & $65(11)$ \\
\hline$C(26)$ & $220(11)$ & $313(12)$ & $275(11)$ & 93(10) & $65(9)$ & $40(10)$ \\
\hline $\mathrm{C}(27)$ & $167(10)$ & $156(10)$ & $182(10)$ & $-12(8)$ & $10(8)$ & 1(8) \\
\hline$C(28)$ & $134(10)$ & $145(10)$ & $164(10)$ & $0(8)$ & $24(8)$ & $34(8)$ \\
\hline C(29) & $174(10)$ & $160(10)$ & $205(10)$ & $-4(8)$ & $36(8)$ & $-8(8)$ \\
\hline$C(30)$ & $237(11)$ & $235(11)$ & 171(10) & $-23(9)$ & $64(9)$ & $16(9)$ \\
\hline $\mathrm{C}(31)$ & $251(12)$ & $187(11)$ & 231(11) & $-67(9)$ & $35(9)$ & $14(9)$ \\
\hline$C(32)$ & 193(11) & 161(11) & $276(12)$ & 11(9) & $38(9)$ & $-11(8)$ \\
\hline$C(33)$ & $176(10)$ & 181(10) & $159(10)$ & $18(8)$ & $44(8)$ & $44(8)$ \\
\hline$C(34)$ & $106(9)$ & $155(10)$ & $155(9)$ & $-13(8)$ & $14(8)$ & $10(8)$ \\
\hline$C(35)$ & $165(10)$ & $183(10)$ & $175(10)$ & 11(8) & $45(8)$ & $-5(8)$ \\
\hline$C(36)$ & 211(11) & $260(12)$ & 161(10) & $-67(9)$ & $46(9)$ & $-39(9)$ \\
\hline$C(37)$ & $176(11)$ & $174(10)$ & $238(11)$ & $-57(9)$ & $30(9)$ & $-26(8)$ \\
\hline $\mathrm{C}(38)$ & $178(11)$ & 184(11) & $232(11)$ & $46(9)$ & $32(9)$ & $-22(9)$ \\
\hline C(39) & $157(10)$ & $221(11)$ & $141(10)$ & $-11(8)$ & $11(8)$ & $8(8)$ \\
\hline$C(40)$ & $150(10)$ & 179(10) & $176(10)$ & $-52(8)$ & $1(8)$ & $16(8)$ \\
\hline$C(41)$ & $255(12)$ & $241(12)$ & $306(12)$ & $0(9)$ & $105(10)$ & $38(9)$ \\
\hline
\end{tabular}




\begin{tabular}{lrrrrrr}
$\mathrm{C}(42)$ & $277(13)$ & $380(14)$ & $410(14)$ & $-67(11)$ & $169(11)$ & $39(11)$ \\
$\mathrm{C}(43)$ & $224(12)$ & $333(13)$ & $440(15)$ & $-134(11)$ & $40(11)$ & $106(10)$ \\
$\mathrm{C}(44)$ & $327(14)$ & $268(13)$ & $393(14)$ & $-15(10)$ & $5(11)$ & $112(10)$ \\
$\mathrm{C}(45)$ & $239(12)$ & $232(12)$ & $252(11)$ & $-10(9)$ & $34(9)$ & $21(9)$ \\
$\mathrm{C}(46)$ & $236(11)$ & $188(11)$ & $186(10)$ & $35(8)$ & $31(9)$ & $-17(9)$ \\
$\mathrm{C}(47)$ & $470(15)$ & $182(11)$ & $321(13)$ & $46(10)$ & $68(11)$ & $-7(10)$ \\
$\mathrm{C}(48)$ & $301(13)$ & $416(14)$ & $272(12)$ & $110(10)$ & $97(10)$ & $-61(11)$ \\
$\mathrm{C}(49)$ & $394(14)$ & $319(13)$ & $191(11)$ & $64(9)$ & $5(10)$ & $-64(10)$ \\
$\mathrm{C}(51)$ & $740(20)$ & $630(20)$ & $565(19)$ & $63(16)$ & $232(17)$ & $92(18)$ \\
$\mathrm{C}(52)$ & $460(18)$ & $590(20)$ & $710(20)$ & $147(16)$ & $49(16)$ & $-84(15)$ \\
$\mathrm{C}(53)$ & $550(20)$ & $391(17)$ & $890(20)$ & $-83(15)$ & $152(18)$ & $28(15)$ \\
$\mathrm{C}(54)$ & $428(18)$ & $376(16)$ & $1050(30)$ & $-40(16)$ & $69(17)$ & $32(13)$ \\
$\mathrm{C}(55)$ & $466(19)$ & $760(20)$ & $750(20)$ & $-256(18)$ & $78(16)$ & $-57(17)$ \\
$\mathrm{C}(56)$ & $522(19)$ & $780(20)$ & $580(19)$ & $7(16)$ & $71(16)$ & $-22(18)$ \\
$\mathrm{C}(61)$ & $293(13)$ & $237(12)$ & $328(13)$ & $39(10)$ & $105(11)$ & $69(10)$ \\
$\mathrm{C}(62)$ & $214(11)$ & $361(13)$ & $228(11)$ & $30(10)$ & $52(9)$ & $69(10)$ \\
$\mathrm{C}(63)$ & $230(12)$ & $309(13)$ & $261(12)$ & $-68(10)$ & $88(10)$ & $-18(10)$ \\
& & & & & \\
\hline
\end{tabular}


Table S9. Hydrogen coordinates $\left(\mathrm{x} 10^{4}\right)$ and isotropic displacement parameters $\left(\AA^{2} \mathrm{x} 10\right.$ 3) for $\left[\mathrm{PhBP}_{3}\right] \mathrm{Co} \equiv \mathrm{N}^{t} \mathrm{Bu}$.

\begin{tabular}{|c|c|c|c|c|}
\hline & $\mathrm{x}$ & $\mathrm{y}$ & $\mathrm{Z}$ & $\mathrm{U}_{\text {iso }}$ \\
\hline $\mathrm{H}(2)$ & 3970 & 1820 & 862 & 22 \\
\hline $\mathrm{H}(3)$ & 4475 & 2704 & 248 & 26 \\
\hline $\mathrm{H}(4)$ & 3068 & 3576 & -133 & 28 \\
\hline $\mathrm{H}(5)$ & 1144 & 3543 & 100 & 29 \\
\hline $\mathrm{H}(6)$ & 645 & 2660 & 709 & 24 \\
\hline $\mathrm{H}(7 \mathrm{~A})$ & 771 & 2209 & 1779 & 18 \\
\hline $\mathrm{H}(7 \mathrm{~B})$ & 2173 & 2373 & 1973 & 18 \\
\hline $\mathrm{H}(8 \mathrm{~A})$ & 897 & 691 & 741 & 17 \\
\hline $\mathrm{H}(8 \mathrm{~B})$ & 85 & 1396 & 767 & 17 \\
\hline $\mathrm{H}(9 \mathrm{~A})$ & 3640 & 1174 & 1759 & 18 \\
\hline $\mathrm{H}(9 \mathrm{~B})$ & 3299 & 785 & 1149 & 18 \\
\hline $\mathrm{H}(11)$ & -326 & 1022 & 3112 & 31 \\
\hline $\mathrm{H}(12)$ & -1335 & 1599 & 3767 & 39 \\
\hline $\mathrm{H}(13)$ & -862 & 2787 & 4043 & 35 \\
\hline $\mathrm{H}(14)$ & 597 & 3411 & 3652 & 32 \\
\hline $\mathrm{H}(15)$ & 1577 & 2849 & 2985 & 29 \\
\hline $\mathrm{H}(17)$ & 2764 & 778 & 3537 & 31 \\
\hline $\mathrm{H}(18)$ & 4663 & 814 & 4103 & 40 \\
\hline H(19) & 6181 & 1520 & 3834 & 35 \\
\hline $\mathrm{H}(20)$ & 5773 & 2206 & 2996 & 29 \\
\hline $\mathrm{H}(21)$ & 3877 & 2176 & 2428 & 24 \\
\hline $\mathrm{H}(22)$ & 3039 & -611 & 2927 & 29 \\
\hline $\mathrm{H}(23)$ & 4683 & -1237 & 3424 & 37 \\
\hline $\mathrm{H}(24)$ & 6503 & -1236 & 3093 & 42 \\
\hline $\mathrm{H}(25)$ & 6669 & -620 & 2250 & 43 \\
\hline $\mathrm{H}(26)$ & 5016 & -17 & 1738 & 32 \\
\hline H(29) & 2903 & -109 & 646 & 21 \\
\hline $\mathrm{H}(30)$ & 2422 & -1049 & -16 & 25 \\
\hline $\mathrm{H}(31)$ & 1414 & -2070 & 228 & 27 \\
\hline $\mathrm{H}(32)$ & 908 & -2169 & 1139 & 25 \\
\hline $\mathrm{H}(33)$ & 1355 & -1227 & 1795 & 20 \\
\hline $\mathrm{H}(35)$ & -761 & 4 & 358 & 21 \\
\hline $\mathrm{H}(36)$ & -1595 & -1085 & -8 & 25 \\
\hline $\mathrm{H}(37)$ & -2206 & -1949 & 596 & 24 \\
\hline $\mathrm{H}(38)$ & -2033 & -1705 & 1576 & 24 \\
\hline $\mathrm{H}(39)$ & -1133 & -634 & 1951 & 21 \\
\hline $\mathrm{H}(41)$ & -1910 & 536 & 2211 & 31 \\
\hline $\mathrm{H}(42)$ & -3639 & 1171 & 2311 & 41 \\
\hline $\mathrm{H}(43)$ & -4163 & 2216 & 1781 & 40 \\
\hline $\mathrm{H}(44)$ & -2971 & 2622 & 1140 & 40 \\
\hline $\mathrm{H}(45)$ & -1258 & 1974 & 1016 & 29 \\
\hline $\mathrm{H}(47 \mathrm{~A})$ & 403 & -1632 & 2782 & 49 \\
\hline $\mathrm{H}(47 \mathrm{~B})$ & 644 & -1782 & 3457 & 49 \\
\hline $\mathrm{H}(47 \mathrm{C})$ & 1697 & -1468 & 3154 & 49 \\
\hline $\mathrm{H}(48 \mathrm{~A})$ & -1191 & -185 & 3310 & 49 \\
\hline $\mathrm{H}(48 \mathrm{~B})$ & -1105 & -983 & 3578 & 49 \\
\hline $\mathrm{H}(48 \mathrm{C})$ & -1354 & -876 & 2897 & 49 \\
\hline $\mathrm{H}(49 \mathrm{~A})$ & 1973 & -431 & 3836 & 46 \\
\hline
\end{tabular}




\begin{tabular}{lrrrr}
$\mathrm{H}(49 \mathrm{~B})$ & 902 & -700 & 4147 & 46 \\
$\mathrm{H}(49 \mathrm{C})$ & 874 & 99 & 3884 & 46 \\
$\mathrm{H}(51)$ & 3959 & 5065 & 9581 & 76 \\
$\mathrm{H}(52)$ & 4579 & 6062 & 10172 & 71 \\
$\mathrm{H}(53)$ & 3158 & 6859 & 10423 & 73 \\
$\mathrm{H}(54)$ & 1114 & 6669 & 10015 & 75 \\
$\mathrm{H}(55)$ & 530 & 5637 & 9506 & 80 \\
$\mathrm{H}(56)$ & 1939 & 4880 & 9202 & 75 \\
$\mathrm{H}(61)$ & 5526 & 8783 & 125 & 34 \\
$\mathrm{H}(62)$ & 6259 & 9624 & 834 & 32 \\
$\mathrm{H}(63)$ & 5723 & 10839 & 714 & 31 \\
\hline
\end{tabular}

